\title{
EL PROCEDIMIENTO DE SENTENCIA PILOTO A EXAMEN: ¿ SUS EFECTOS SOBRE EL SISTEMA EUROPEO DE PROTECCIÓN DE DERECHOS HUMANOS SON TRANSFORMADORES, BENEFICIOSOS O PERVERSOS'?
}

\author{
YAELLE CACHO SÁNCHEZ \\ Universidad de Cantabria \\ yaelle.cacho@unican.es
}

Cómo citar/Citation

Cacho Sánchez, Y. (2020)

El procedimiento de sentencia piloto a examen: ¿̇sus efectos sobre el sistema europeo de protección de derechos humanos son transformadores, beneficiosos o perversos? Revista de Derecho Comunitario Europeo, 65, 121-163. doi: https://doi.org/10.18042/cepc/rdce.65.04

\section{Resumen}

El presente trabajo, centrado en el procedimiento de sentencia piloto, tiene como propósito principal evaluar el impacto de este instrumento en el sistema europeo de protección de derechos humanos. Para ello, en primer lugar, identifica los rasgos principales de este procedimiento en su configuración actual, teniendo en cuenta que ha sido creado y desarrollado por la vía pretoriana en el contexto del proceso de reforma del Convenio Europeo de Derechos Humanos. Su estudio nos ha dado la ocasión para apreciar que el "problema sistémico» habilitante del procedimiento

1 Este trabajo se realiza en el marco de los proyectos «European Union in the World: International Law and Politics», Erasmus + Programme-Jean Monnet Activities, Ref. 587791-EPP-1-2017-1-ES-EPPJMO-MODULE y «Judicialización y diálogo judicial europeo e internacional», UC, Ref. 07.P148.64004. 
de sentencia piloto no parece responder a los mismos parámetros que la noción de «deficiencia sistémica» utilizada por la Unión Europea. En segundo lugar, se ha abordado el análisis de los efectos que el procedimiento de sentencia piloto provoca en la relación del TEDH con los demandantes, con el Estado demandado y con el Comité de Ministros. Todo ello nos permite concluir que es un procedimiento ciertamente transformador, que ya está desplegando interesantes efectos beneficiosos, pero que también exige reajustes a fin de evitar sus preocupantes efectos perversos sobre el sistema.

\title{
Palabras clave
}

Convenio Europeo de Derechos Humanos; Tribunal Europeo de Derechos Humanos; procedimiento de sentencias piloto; proceso de reforma del CEDH; derecho de recurso individual; ejecución de sentencias; problema sistémico.

\section{THE PILOT-JUDGMENTS PROCEDURE UNDER SCRUTINY: ARE ITS EFFECTS ON THE EUROPEAN SYSTEM OF HUMAN RIGHTS PROTECTION TRANSFORMATIVE, BENEFICIAL OR PERVERSE?}

\begin{abstract}
The following paper focused on the pilot judgment procedure aims to evaluate the impact of this instrument on the European Convention on Human Rights system. First of all, this has required to identify the main characteristics of this procedure in its current form, taking into account that it has been created and developed by jurisprudence in the context of the ECHR reform process. This provided us an opportunity to observe that there is not a perfect correlation between the «systemic problem» in the pilot judgment procedure and the notion of «systemic deficiencies» used by the EU Court of Justice. Secondly, the effects of the pilot judgment procedure in the relations between the European Court of Human Rights with the applicants, with the respondent state and with the Committee of Ministers have been analysed. As a result, we are facing a certain transformative procedure, which is deploying some beneficial effects on the Convention system, but which also requires several adjustments in order to avoid its perverse effects.
\end{abstract}

\section{Keywords}

European Convention on Human Rights; European Court of Human Rights; pilot judgment procedure; ECHR reform process; access to justice; execution; systemic problem. 


\section{LA PROCÉDURE DE L'ARRÊT PILOTE SOUS EXAMEN: SES EFFETS SUR LE SYSTĖME EUROPÉEN DES DROITS DE L'HOMME, SONT-ILS TRANSFORMATEURS, BÉNÉFIQUES OU PERVERS?}

\section{Résumé}

La présente étude axé sur la procédure d'arrêt pilote cherche à évaluer l'impact de cet instrument sur le système de la Convention européenne des droits de l'homme. Cela a exigé tout d'abord d'identifier les principales caractéristiques de cette procédure sous sa forme actuelle, tenant compte du fait qu'elle a été créée et développée par la voie prétorienne dans le contexte de la réforme de la Convention. Son étude nous a permis d'observer qu'il n'y a pas une parfaite corrélation entre le «problème systémique» de la procédure d'arrêt pilote et la notion de «défaillances systémiques» utilisée para la Cour de justice de l'Union européenne. Ensuite, il a fallu analyser les effets que la procédure d'arrêt pilote entraîne sur les relations de la Cour européenne des droits de l'homme avec les requérants, avec l'État défendeur et avec le Comité des Ministres. Tout cela nous a permis de conclure que nous nous trouvons aujourd'hui devant une procédure certainement transformatrice, qui est déjà en train de déployer des effets bénéfiques sur le système de la Convention, mais qui exige aussi certains réajustements afin d'éviter ses redoutables effets pervers.

\section{Mots clés}

Convention européenne des droits de l'homme; Cour européenne des droits de l'homme; procédure d'arrêt pilote; réforme de la Cour européenne des droits de l'homme; droit de recours individuel; exécution des arrêts; problème systémique. 


\section{SUMARIO}

I. INTRODUCCIÓN. II. EL PROCEDIMIENTO DE SENTENCIA PILOTO: 1. La decisión de tramitar una demanda como piloto. 2. La sentencia piloto. 3. La ejecución de la sentencia piloto. III. LOS EFECTOS DEL PROCEDIMIENTO DE SENTENCIA PILOTO EN EL SISTEMA DEL CEDH: 1. Los demandantes «piloto» y los demandantes «clones»: el derecho de recurso individual ante el TEDH. 2. La relación entre el Estado demandado y el TEDH: el principio de subsidiariedad. 3. El reparto de competencias entre el Comité de Ministros y el TEDH: el control de la ejecución de las sentencias piloto. IV. CONCLUSIONES. BIBLIOGRAFía.

\section{INTRODUCCIÓN²}

El procedimiento de sentencia piloto es una de las medidas adoptadas en el marco del proceso de reforma del sistema de protección del Convenio Europeo de Derechos Humanos (CEDH) para contribuir a la resolución de uno de los problemas que se identificaron inicialmente como más graves: el gran número de demandas individuales que tenían la misma causa estructural a nivel interno (los contenciosos repetitivos) ${ }^{3}$. Nace, por tanto, para ayudar al TEDH a gestionar su carga de trabajo con mayor eficacia, pero también a los Estados a resolver los problemas sistémicos o estructurales a nivel nacional ${ }^{4}$, identificándose posteriormente un tercer objetivo: ofrecer a los individuos afectados

2 Toda la documentación utilizada está disponible en el Portal del Consejo de Europa (www.coe.int), en particular en la página web del Tribunal Europeo de Derechos $\mathrm{Hu}$ manos (www.echr.coe.int) y en su base de datos HUDOC (https://hudoc.echr.coe. int). Todas las páginas web de referencia han sido consultas por última vez el 24 de octubre de 2019.

3 Rapport explicatif, Protocole no 14 à la Convention de sauvegarde des Droits de l'Homme et des Libertés fondamentales, amendant le système de contrôle de la Convention, párrs. 7 y 8 .

4 Broniowski c. Pologne (au principal) [GC], n 31443/96, CEDH 2004 V, párrs. 190, 191 y 194; Burmych et autres c. Ukraine [GC], $\mathrm{n}^{\text {os }} 46852 / 13$ et al., arrêt (radiation), 12 de octubre de 2017, párr. 158. 
una reparación más rápida ${ }^{5}$. Sobre la base de este triple objetivo, se configura un procedimiento que permite al TEDH identificar disfunciones crónicas en un Estado e indicarle las medidas correctoras que deberá adoptar en el ámbito nacional para hacerles frente y para lograr una solución que pueda extenderse más allá del caso concreto, a todas las posibles víctimas ${ }^{6}$.

El procedimiento de sentencia piloto no fue, sin embargo, incorporado en el Protocolo $\mathrm{n}^{\circ} 14^{7}$, sino que surge por la vía pretoriana sobre la base de una interpretación renovada del art. $46 \mathrm{CEDH}$. En respuesta a la invitación del Comité de Ministros del Consejo de Europa cursada el día antes de la firma de aquel protocolo para que actuara frente a los contenciosos repetitivos ${ }^{8}$, el Tribunal Europeo de Derechos Humanos (TEDH) dictó la primera sentencia piloto en el asunto Broniowski c. Polonia [GS]. Esta rápida reacción del tribunal parece que podría perseguir boicotear una reforma que sí incorporó el Protocolo no 14 al art. 46.3, 4 y 5 CEDH, que suponía atribuir al Comité de Ministros la capacidad para iniciar dos tipos de procedimientos ante el TEDH cuando el Estado no esté dispuesto a ejecutar una sentencia o sea incapaz por una falta de claridad de la misma (Szymczak, 2011: 283).

A pesar de que en 2011 se modificó el Reglamento de Procedimiento del TEDH a los efectos de su regulación, el procedimiento de sentencia piloto se ha seguido desarrollando a través de la jurisprudencia del TEDH, lo que le ha dotado de gran flexibilidad y facilidad de adaptación. El art. 61 del

5 TEDH, Fiche thématique - Les arrêts pilotes, Unité de Presse, enero 2019, p. 1; Di Marco, 2016: 889; Leach et al., 2010b: 13; Queralt Jiménez, 2018: 406. Esta evolución en los objetivos es subrayada por Buyse, 2016: 108.

6 Burmych et autres c. Ukraine, cit., párr. 159.

7 Protocole $n^{\circ} 14$ à la Convention de sauvegarde des Droits de l'Homme et des Libertés fondamentales, amendant le système de contrôle de la Convention (STCE $\left.n^{\circ} 194\right)$ ), Strasbourg, 13 de mayo de 2004, que España ratificó el 15 de marzo de 2006 y que entró en vigor el 1 de junio de 2010 (BOE, núm. 130, de 28 de mayo de 2010, pp. 46324 a 46335). Aunque el Protocolo no 14 no dice nada al respecto, su Informe explicativo menciona expresamente que sería útil que "la Cour et, en ce qui concerne la surveillance de l'exécution des arrêts, le Comité des Ministres adoptent une procédure spéciale afin de traiter de façon prioritaire les arrêts qui démontrent un problème structurel capable de générer un nombre significatif de requêtes répétitives, afin que l'arrêt soit exécuté rapidement» (Rapport explicatif, Protocole $n^{\circ} 14$ à la Convention ..., cit., párr. 16).

$8 \mathrm{CM} / \operatorname{Res}(2004) 3$, Resolution of the Committee of Ministers on judgments revealing an underlying systemic problem, 12 de mayo de 2004, párr. 1. En la misma sesión, el Comité de Ministros adoptó una segunda resolución dirigida a los Estados sobre este mismo particular (Resolution Res(2004)6 of the Committee of Ministers to member states on the improvement of domestic remedies, 12 de mayo de 2004). 
Reglamento se limitó a codificar la praxis existente hasta ese momento, reducida a unas quince sentencias piloto dictadas en su mayoría a partir de 2009 que, en consecuencia, se encontraban en 2011 en una fase temprana de ejecución. Esta disposición no contempla, por tanto, los interesantes desarrollos que se vienen produciendo desde entonces, en particular, en la fase de ejecución, que además se han visto impulsados por el cambio de paradigma en la definición del principio de subsidiariedad sobre el que precisamente se funda este procedimiento a resultas del proceso de Interlaken, lo que ha tenido su reflejo en la evolución y alcance de sus efectos.

Dado que el largo proceso de reforma del sistema de protección del CEDH se encuentra en fase de conclusión ${ }^{9}$, lo que nos permite además tener una visión de conjunto del mismo, y puesto que contamos ya con un total de 35 procedimientos de sentencia piloto ${ }^{10}$, parece oportuno someter al procedimiento de sentencia

9 Conférence de haut niveau sur l'avenir de la Cour européenne des droits de l'homme, Déclaration d'Interlaken, 19 de febrero de 2010, Plan de acción, párrs. 3 a 6; CM, Rapport sur les moyens de garantir l'efficacité continue du système de la Convention européenne des droits de l'homme, 129e Session du Comité des Ministres (Helsinki, 16-17 mai 2019), Doc. CM(2019)70-final, párr. 47.

10 En el asunto Burmych et autres c. Ukraine (cit., párr. 162), el propio TEDH menciona que ha dictado 35 sentencias piloto, aunque luego solo cita expresamente los 33 asuntos siguientes (que listamos por orden alfabético): Alisić et autres c. Bosnie-Herzégovine, Croatie, Serbie, Slovénie et l'ex-République yougoslave de Macédoine [GC], n 60642/08, CEDH 2014; Ananyev et autres c. Russie, $\mathrm{n}^{\text {os }} 42525 / 07$ et 60800/08, 10 janvier 2012; Broniowski c. Pologne (au principal) [GC], n 31443/96, CEDH 2004 V; Bourdov c. Russie ( $\left.n^{\circ} 2\right), \mathrm{n}^{\circ} 33509 / 04, \mathrm{CEDH}$ 2009; Dimitrov et Hamanov c. Bulgarie, $\mathrm{n}^{\text {os }} 48059 / 06$ et 2708/09, 10 de mayo de 2011; Finger $c$. Bulgarie, $\mathrm{n}^{\circ}$ 37346/05, 10 de mayo de 2011; Gazsó c. Hongrie, n 48322/12, 16 de julio de 2015; Gerasimov et autres c. Russie, $\mathrm{n}^{\circ}$ 29920/05 et dix autres requêtes, 1 de julio de 2014; Glykantzi c. Grèce, n 40150/09, 30 de octubre de 2012; Greens et M.T. c. Royaume-Uni, $\mathrm{n}^{\text {os }} 60041 / 08$ et 60054/08, CEDH 2010 (extraits); Hutten Czapska c. Pologne [GC], n 35014/97, CEDH 2006 VIII; Kauczor c. Pologne, $\mathrm{n}^{\circ}$ 45219/06, 3 de febrero de 2009; Kurić et autres c. Slovénie [GC], n 26828/06, CEDH 2012 (extraits); Lukenda c. Slovénie, n 23032/02, CEDH 2005 X; M.C. et autres c. Italie, $\mathrm{n}^{\circ}$ 5376/11, 3 de septiembre de 2013; Manushaqe Puto et autres $c$. Albanie, $\mathrm{n}^{\text {os }}$ 604/07 et 3 autres, 31 de julio de 2012; Maria Atanasiu et autres c. Roumanie, $\mathrm{n}^{\text {os }} 30767 / 05$ et 33800/06, 12 de octubre de 2010; Michelioudakis c. Grèce, $\mathrm{n}^{\circ} 54447 / 10,3$ de abril de 2012; Neshkov et autres c. Bulgarie, $\mathrm{n}^{\text {os }} 36925 / 10$ et 5 autres requêtes, 27 de enero de 2015; Norbert Sikorski c. Pologne, $\mathrm{n}^{\circ}$ 17599/05, 22 de octubre de 2009; Olaru et autres c. Moldova, $\mathrm{n}^{\text {os }}$ 476/07 et 3 autres, 28 de julio de 2009; Orchowski c. Pologne, $\mathrm{n}^{\circ}$ 17885/04, 22 de octubre de 2009; Rezmiveş et autres c. Roumanie, $\mathrm{n}^{\text {os }}$ 61467/12 et 3 autres, 25 de abril de 2017; Rumpf c. Allemagne, $\mathrm{n}^{\circ}$ 46344/06, 2 de septiembre de 2010; Rutkowski et autres $c$. Pologne, $\mathrm{n}^{\text {os }} 72287 / 10$ et 2 autres, 7 de julio 
piloto a examen. En particular, puede preguntarse por el impacto que está teniendo en el sistema de protección del CEDH desde una doble perspectiva. Se trata de averiguar si está respondiendo efectivamente a los objetivos para los que fue creado y si provoca algún efecto adicional sobre el sistema de protección del CEDH.

Con este fin, nuestro trabajo comienza con un primer epígrafe dedicado a la identificación de los rasgos principales del procedimiento de sentencia piloto considerado en su conjunto y que lo configuran como un instrumento novedoso (II). En segundo lugar, analizaremos los efectos que estos elementos innovadores provocan en la relación del TEDH con los demandantes, los Estados demandados y el Comité de Ministros (III). De este modo, esperamos verificar nuestra hipótesis de partida: el procedimiento de sentencia piloto ha cumplido los objetivos para los que fue creado, especialmente enfrentar la sobrecarga de trabajo del TEDH, pero en su configuración actual podría acabar generando importantes riesgos en el sistema del CEDH a largo plazo.

\section{EL PROCEDIMIENTO DE SENTENCIA PILOTO}

Si bien es cierto que es posible reconocer algunos de los elementos principales que caracterizan al procedimiento de sentencia piloto en la

de 2015; Scoppola c. Italie ( $n^{\circ}$ 2) [GC], $\mathrm{n}^{\circ}$ 10249/03, 17 de septiembre de 2009; Suljagić c. Bosnie-Herzégovine, $\mathrm{n}^{\circ}$ 27912/02, 3 de noviembre de 2009; Torreggiani et autres c. Italie, $\mathrm{n}^{\text {os }} 43517 / 09$, et six autres requêtes, 8 de enero de 2013; Ümmühan Kaplan c. Turquie, $\mathrm{n}^{\circ}$ 24240/07, 20 de marzo de 2012; Varga et autres c. Hongrie, $\mathrm{n}^{\text {os }}$ 14097/12 et cinq autres requêtes, 10 de marzo de 2015; Vassilios Athanasion et autres $c$. Grèce, $\mathrm{n}^{\circ}$ 50973/08, 21 de diciembre de 2010; W.D. c. Belgique, $\mathrm{n}^{\circ}$ 73548/13, 6 de septiembre de 2016; Xenides-Arestis c. Turquie, $\mathrm{n}^{\circ}$ 46347/99, 22 de diciembre de 2005; Youri Nikolayevich Ivanov c. Ukraine, no 40450/04, 15 de octubre de 2009. Los otros dos asuntos piloto no mencionados podrían ser: Scordino c. Italia ( $\left.n^{\circ} 1\right)[G C], \mathrm{n}^{\circ} .36813 / 97,29$ de marzo de 2006 - que el propio tribunal menciona como tal en el asunto Varga y otros (cit., párr. 49) (confirman esta identificación autores como Abrisketa Uriarte, 2013: 82; Buyse, 2016: 106; Garlicki, 2007: 189; Sardurski, 2009: nota 118; Szymczak, 2011: 279 y 282) - y Doğan and Others v. Turkey, no 8803/02 and 14 more, Judgment (Merits), ECHR 2004-VI — que si bien no fue considerado un procedimiento piloto en la sentencia original, el TEDH le reconoció esta naturaleza con carácter retroactivo, al tomar la decisión de inadmitir el asunto İ́gyer c. Turquie, no 18888/02, adm. dec, $12 \mathrm{de}$ enero de 2006, párrs. 73 y 94 (confirman esta identificación autores como Buyse, 2016: 106). No hemos encontrado ninguna referencia posterior a ningún procedimiento de sentencia piloto, ni siquiera en HUDOC. 
jurisprudencia del TEDH anterior al nacimiento de este instrumento (Haider, 2013: 16-23; Wildhaber, 2009: 69), la novedad radica en presentarse juntos, y en conjunción con otros, lo que les ha permitido evolucionar para configurar un nuevo instrumento a disposición del TEDH. El análisis de estos elementos requiere partir del art. 61 del Reglamento del TEDH, aunque es preciso realizar dos precisiones al respecto. En primer lugar, las quince primeras sentencias piloto adoptadas hasta 2011 no respondieron en todos los casos a un modelo uniforme, pero contribuyeron a la consolidación de una práctica que finalmente quedó plasmada en el art. 61 del Reglamento. Esta falta de uniformidad ha dificultado la identificación de algunas de estas primeras sentencias como tales, mientras el TEDH no se ha pronunciado específicamente al respecto ${ }^{11}$.

En segundo lugar, como vimos, en 2011 estas primeras sentencias piloto se encontraban en su mayoría en una fase temprana de ejecución, por lo que las previsiones del art. 61 al respecto son escasas. El análisis de los elementos principales que caracterizan al procedimiento de sentencia piloto requiere, por tanto, completar el examen de esta disposición con un estudio de la práctica posterior. Este análisis se ordenará siguiendo las tres grandes fases en que queda estructurado este procedimiento de conformidad con el Reglamento del Tribunal: a) la decisión de tramitar una demanda siguiendo el procedimiento de sentencia piloto, b) la adopción de una sentencia piloto, y c) la fase de ejecución, que culminará, en su caso, con el cierre del procedimiento (art. 61.10).

\section{LA DECISIÓN DE TRAMITAR UNA DEMANDA COMO PILOTO}

De conformidad con el art. 61 del Reglamento, el TEDH (tanto en su formación de Gran Sala como de Sala), de oficio o a instancia de parte, y una vez oídas las partes, es quien únicamente tiene la capacidad para aplicar el procedimiento de sentencia piloto a una demanda ${ }^{12}$. Una vez tomada la decisión,

11 Véase la nota 8. Esta situación también contribuyó a que la doctrina se esforzase por definir los elementos que debían tener las sentencias piloto, diseñando algunos modelos ideales (Wildhaber, 2009: 71; Garlicki, 2007: 185; Leach et al., 2010b: 22; Fyrnys, 2011: 1232 y 1233; Haider, 2013: 36). Dependiendo del nivel de adecuación a aquellos modelos, desarrollaron diferentes categorizaciones, como la que distingue entre: «full pilot judgments», «quasi-pilot judgments» $y$ "other judgment addressing systemic issues» (Leach et al., 2010b: 172 y 173).

12 Reglamento TEDH, art. 61.2.b). Así, por ejemplo, en el caso Cordella y otros c. Italia, el TEDH decidió que no procedía iniciar el procedimiento de sentencia piloto a pesar de que los demandantes lo habían solicitado (TEDH, Cordella et autres c. Italie, 
la tramitación de la demanda tendrá carácter prioritario ${ }^{13}$. Para adoptar esta decisión tiene, no obstante, que haber constatado la concurrencia del supuesto habilitante: la demanda presentada ante él debe revelar la existencia en el Estado demandado de «un problema estructural o sistémico u otra disfunción similar que haya dado lugar o sea susceptible de dar lugar a la introducción de otras demandas análogas» (art. 61.1).

Sin embargo, el art. 61 no ofrece una definición sobre lo que debe entenderse por "problema estructural o sistémico u otra disfunción similar». Por su parte, el tribunal no suele motivar detalladamente su decisión de aplicar el procedimiento (Glas, 2016: 46 y 49); incluso ha empleado estos términos indiferentemente para designar el mismo fenómeno (Lambert Abdelgawad, 2007: 362; Susi, 2012: 398), aunque en algunas ocasiones se ha detenido en el examen de la causa del problema (Haider, 2013: 43). Por otro lado, en muchos casos la identificación de un problema sistémico o estructural no ha llevado al TEDH a tomar la decisión de iniciar un procedimiento de sentencia piloto $^{14}$. En ocasiones, incluso, el tribunal ha tomado esta decisión después de que el Comité de Ministros hubiera hecho esa calificación (Leach et al., 2010a: 22) o después de haber dictado diferentes sentencias condenando al Estado demandado en casos particulares por el mismo problema sistémico o estructural ${ }^{15}$. En estas ocasiones, el tribunal parece querer dejar tiempo al

$\mathrm{n}^{\text {os }} 54414 / 13$ y 54264/15, Sentencia del 24 de enero de 2019, párrs. 177 y 180). Tampoco se exige el consentimiento del Estado, aunque los casos más exitosos han contado con al menos un aliado dentro del Estado - por ejemplo, de los tribunales constitucionales nacionales en contra del ejecutivo o legislativo nacional- (Leach et al., 2010a: 177; Sadurski, 2009: 22, 27 y 28), por lo que el apoyo del Estado o en el Estado debiera ser un elemento relevante a valorar por el tribunal al adoptar su decisión.

13 Reglamento TEDH, arts. 61.2.c). En particular, el TEDH ha catalogado las demandas objeto de un procedimiento de sentencia piloto como asuntos de categoría II a efectos de prioridad, teniendo en cuenta que ha establecido siete categorías y que la categoría I (que comprende las demandas urgentes que afectan a demandantes vulnerables) es de máxima prioridad. Véase TEDH, La politique de priorisation de la Cour, 22 de mayo de 2017.

14 Así, por ejemplo, en el reciente asunto Petukhov v. Ukraine (no 2), no 41216/13, Sentencia de 12 de marzo de 2019, párrs. 192 y 194.

15 Así, por ejemplo, al tiempo de pronunciarse sobre el caso Youri Nikolayevich Ivanov c. Ucrania, el TEDH ya había dictado contra Ucrania unas trescientas sentencias sobre el mismo problema desde 2004 (cit., párrs. 83 y 86); en el asunto Greens y M.T. c. Reino Unido, la sentencia piloto se dictó cinco años después de la primera sentencia sobre el mismo problema sistémico (Hirst $\left(n^{o} 2\right)$ c. Reino Unido de 6 de octubre de 
Estado para que remedie la situación, tomando la decisión de iniciar un procedimiento de sentencia piloto solo cuando no se alcanza este resultado de forma satisfactoria (Susi, 2012: 413).

Si bien algún autor, como la antigua juez y vicepresidenta del TEDH Tulkens, se centra en distinguir ambos conceptos, en particular atendiendo al origen de la disfunción y en consecuencia al diferente tipo de medidas generales que requieren ${ }^{16}$, lo cierto es que la diferencia puede ser muy sutil en la práctica $^{17}$. Tal vez por ello, no solo el TEDH, sino también la mayoría de la doctrina usa en muchos casos ambos conceptos indistintamente (aunque no necesariamente sean intercambiables) y no se centra en las diferencias entre ellos (Czepek, 2018: 351). Es más, es poco habitual encontrar trabajos centrados en la definición que hace el TEDH de los términos "problema estructural o sistémico", salvo contadas excepciones (como Susi, 2012). Si bien un tratamiento pormenorizado de esta cuestión nos distraería de nuestro objetivo, parece, no obstante, oportuno traer a colación dos precisiones al respecto por su contribución a una mejor contextualización y comprensión del procedimiento de sentencia piloto.

En primer lugar, un "problema sistémico o estructural» es un problema que, al traer causa en una deficiencia en el sistema legal o en una práctica

2005); la sentencia piloto en el caso Burdov c. Rusia (no 2) c. Rusia no se adoptó hasta después de unas doscientas sentencias (párr. 129); y en el asunto W. D., el primer pronunciamiento del TEDH se dictó cuatro años después del caso $L$. B. c. Bélgica, de 22 de septiembre de 2012 (párr. 166).

16 Para Tulkens, una situación «sistémica» «affects a large number of people and stems or results not just an act or omission by a State Party but from a defective legal provision or a widespread administrative practice and which, in turn, points to legislation changes needed to resolve the problem, with accompanying administrative and budgetary measures» (como en el asunto Broniowski c. Polonia); mientras que una disfunción «estructural» «concerns mostly the organization or regime», ya que el problema es «the result of a structural breakdown, usually due to lack of sufficient resources, or inefficient organisation. Remedial measures in this context are more dependent on budgetary means» (como, por ejemplo, en el asunto Burdov c. Rusia $n^{\circ} 2$ ). Véase Tulkens, F., «Perspectives from the Court. A typology of the pilot-judgment procedure», en Responding to systemic human rights violations. Pilot judgments of the European Court of Human Rights and their impact at national level, Seminar Strasbourg, 14 June 2010, 1-2, 5 (obtenido de Czepek, 2018: 352 y 353)

17 Como señala Czepek (2018: 354), no siempre es fácil la identificación del origen de la disfunción, como, por ejemplo, en supuestos de duración excesiva del procedimiento, que pueden traer causa en un problema de organización judicial («estructural») y/o en una deficiencia legislativa («sistemática»). 
nacional contrarias al CEDH, va más allá de un caso aislado, de un «simple episodio», de una "violación puntual». Se caracteriza, por tanto, por su capacidad potencial para generar un número considerable de personas afectadas que podrían plantear demandas ante el TEDH, es decir, por su capacidad potencial para provocar contenciosos repetitivos ${ }^{18}$. Como vimos, con el fin de afrontar eficazmente esta problemática, el procedimiento de sentencia piloto nace como un instrumento para superar el problema de las sentencias adoptadas caso por caso (Susi, 2012: 391), permitiendo al TEDH lograr una solución que pueda extenderse más allá del caso concreto, evitando innecesarias repeticiones para alcanzar la misma conclusión en un amplio número de casos y, de este modo, gestionar su carga de trabajo con mayor eficacia.

Sobre todo en las primeras sentencias piloto, el TEDH mencionaba expresamente datos concretos sobre el considerable número de asuntos pendientes o potenciales similares ${ }^{19}$ o de sentencias previas que traían la misma causa contra un Estado $^{20}$ (Glas, 2016: 46; Susi, 2012: 410). Sin embargo, el Reglamento no incorporó la exigencia de que la disfunción deba generar efectivamente un gran número de sentencias o de demandas análogas ante el tribunal, ni llegar a generar un elevado número de demandas potenciales. Los datos podrían considerarse, en su caso, como indicadores reveladores de la existencia de la disfunción. El conjunto de personas afectadas (demandantes actuales o potenciales) tampoco tiene que ser una colectividad homogénea. Se puede identificar un colectivo específico cuando el problema es relativamente concreto $^{21}$, pero en otros casos la categoría de personas afectadas puede ser

18 Véase, a modo de ejemplo, CM, Résolution (2004)3 sur les arrêts qui révèlent un problème structurel sous-jacent»; Hutten-Czapska c. Polonia, cit., párr. 236; Déclaration d'Interlaken, Plan d'Action, D; o Asamblea Parlamentaria, Résolution 1914 (2013), Assurer la viabilité de la Cour de Strasbourg: les insuffisances estructurelles dans les Etats Parties, 22 de enero de 2013, párr. 3.

19 Así, por ejemplo, en el asunto Broniowski c. Polonia, el TEDH menciona que el número de demandas pendientes era de 167 y que los demandantes potenciales ascendían a 80000 aproximadamente (cit., párr. 193); igualmente en el asunto Hutten-Czapska c. Polonia, los datos que se manejaban era de 18 demandas pendientes (párr. 236) y unos 100000 propietarios y entre 600000 y 900000 inquilinos (cit., párr. 235).

20 Atanasiu y otros c. Rumanía, cit., párr. 215; Burdov c. Rusia (no 2), cit., párr. 129; Youri Nikolayevich Ivanov c. Ucrania, cit., párr. 83.

21 Como, por ejemplo, en el asunto Hutten-Czapska $c$. Polonia, en que la disfunción se vinculaba a la legislación reguladora del alquiler de inmuebles que perjudicaba a los derechos de los propietarios. 
excesivamente abierta e indeterminada al incluir una variedad de situaciones $^{22}$ (Abrisketa Uriarte, 2013: 82; Leach et al., 2010b: 351).

En segundo lugar, un "problema sistémico o estructural», de este modo definido en el seno del Consejo de Europa, por muy importante o grave que sea la disfunción observada en el orden jurídico nacional, no necesariamente ocasiona una "violación grave de los derechos humanos» ${ }^{23}$, y mucho menos

22 Por ejemplo, en el asunto Burdov c. Rusia ( $n^{\circ} 2$ ), en que la no ejecución de las sentencias adoptadas por los tribunales nacionales rusos afectó a víctimas de la catástrofe de Chernóbil, pero también a otras 700 en situaciones dispares. Las situaciones eran tan distintas que del procedimiento piloto Burdov c. Rusia ( $\left.n^{\circ} 2\right)$ acabó desgajándose el procedimiento Gerasimov y otros c. Rusia.

23 De hecho, por ejemplo, en su Resolución 1787 (2011), la Asamblea Parlamentaria, después de mostrar su preocupación por «la persistance de déficiences systématiques majeures qui sont à l'origine de beaucoup de constats répétitives de violations de la Convention» (párr. 5), menciona las diferentes disfunciones de este tipo identificadas en diez Estados miembros, calificando únicamente como "violations graves et répétées des droits de l'homme» la acción de las fuerzas de seguridad rusas en la República de Chechenia (párr. 7.7). Es importante recordar en este punto que el término «violación grave de los derechos humanos» (aunque a veces también se utiliza "violaciones manifiestas» o «violaciones flagrantes» como sinónimos), cuyo origen se situaría en 1967 en el marco de las acciones adoptadas por la ONU respecto de la política del apartheid y de discriminación racial y de segregación en determinados países, está consolidado en la actualidad como categoría jurídica con significado autónomo (por ejemplo, J. P. Albán Alencastro, Las Graves Violaciones a los Derechos Humanos como categoría jurídica, Pro homine [blog], 3 noviembre 2013, disponible en: <http://prohomine. wordpress.com/2013/11/03/las-graves-violaciones-a-losderechos-humanos-como-categoria-juridica/ $>$ ). Cierto es que no contamos con una definición de lo que debe entenderse por «violación grave de los derechos humanos». Se ha dicho que se trataría de violaciones que "por su carácter muy grave constituyen una afrenta a la dignidad humana" (Principios y directrices básicos sobre el derecho de las víctimas de violaciones manifiestas de las normas internacionales de derechos humanos y de violaciones graves del derecho internacional humanitario a interponer recursos y obtener reparaciones, Resolución 60/147 de la Asamblea General, de 16 de diciembre de 2005, anexo, Preámbulo, párr. 6), lo que nos remite a estándares valorativos comunes, a una especie de conciencia jurídica universal, que no deja de ser un concepto evolutivo, de ahí que su determinación concreta se haya producido mayormente a través de la práctica y de la jurisprudencia. Han contribuido a su conceptualización y siguen haciéndolo los mecanismos convencionales y extraconvencionales de los sistemas universal y regionales de protección de derechos humanos, pero también los trabajos de la Comisión de Derecho Internacional de las Naciones Unidas y los desarrollos en ámbitos como la Responsabilidad internacional del Estado o el Derecho Internacional Penal. 
una violación «sistemática» (cometida de conformidad con un plan o política preconcebida) o "generalizada» (cometida a gran escala) que pudiera llegar a ser constitutiva de un "crimen internacional» ${ }^{24}$. Esta interpretación no solo es lógica con el origen del procedimiento de sentencia piloto y con los objetivos que persigue, sino que también resulta evidente a la luz de las dos consideraciones siguientes.

Por un lado, en la política de priorización del TEDH, el procedimiento de sentencia piloto queda englobado entre los «asuntos que plantean cuestiones susceptibles de tener una incidencia sobre la eficacia del sistema del Convenio», equiparándolo con un "problema estructural o situación endémica que el TEDH no ha tenido todavía la oportunidad de examinar». Y se distingue de los «asuntos urgentes», de los «asuntos que plantean una cuestión

24 Véase el umbral de gravedad que han de alcanzar los crímenes internacionales, en particular los crímenes de lesa humanidad, por ejemplo, en Cassesse, A. Crimes against Humanity. En A. Cassesse, et al. (eds.) (2002), The Rome Statute of the International Criminal Court. A Commentary, Oxford, pp. 353-378, y Márquez Carrasco, C. (2008). Los elementos comunes de los crímenes contra la humanidad en Derecho Internacional Penal, Revista General de Derecho Penal, 9. Debe tenerse en cuenta que los crímenes internacionales comprenden las más graves violaciones de los derechos humanos, pero que existen otras violaciones graves de los derechos humanos que no tienen esta consideración pese a alcanzar un cierto umbral de gravedad, al no presentar los elementos constitutivos de estos crímenes. En este sentido, por ejemplo, el Consejo de Derechos Humanos de las Naciones Unidas (cuyo mandato es «ocuparse de las situaciones en que se violen los derechos humanos, incluidas las violaciones graves y sistemáticas», que no «sistémicas» — Resolución 60/251 de la Asamblea General, de 15 de marzo de 2006, párr. 3-) condenó «las violaciones sistemáticas, generalizadas y graves de los derechos humanos que el Gobierno de Eritrea ha cometido y sigue cometiendo en un clima de impunidad generalizada" (Doc. A/HCR/RES/29/18, de 2 de julio de 2015, párr. 10 del Preámbulo y párr. dispositivo 3), condenando de manera separada «las ejecuciones extrajudiciales, las torturas y los malos tratos, incluidos la violencia y abusos de carácter sexual, al servicio nacional y al trabajo forzoso [que] podrían constituir crímenes de lesa humanidad» (párr. 10 del Preámbulo; párr. dispositivo 4). También ha calificado determinadas situaciones de violaciones graves (en Siria en Doc. A/HRC/RES/S-18/1, 2 de diciembre de 2011, Preámbulo, párr. 5) o sistemáticas (en la República Bolivariana de Venezuela, Doc. A/HCR/RES/42/25, de 27 de septiembre de 2019, párr. 6 del Preámbulo) de todos los derechos humanos (civiles y políticos, pero también económicos sociales y culturales). Los Estados que cometan estas violaciones graves y sistemáticas de los derechos humanos pueden ver suspendidos sus derechos inherentes a formar parte del Consejo de Derechos Humanos por una decisión de la Asamblea General adoptada por mayoría de dos tercios de los miembros presentes y votantes (Doc. A/RES/60/251, párr. 8). 
relevante de interés general (especialmente una cuestión grave susceptible de tener repercusiones de consideración sobre los sistemas jurídicos nacionales o sobre el sistema europeo)» y de los «asuntos repetitivos, o no, que comprenden prima facie quejas principales respecto de los arts. 2, 3, 4 o $5 \$ 1$ del Convenio (los derechos más fundamentales, core rights) y que han dado lugar a amenazas directas para la integridad física y la dignidad de la persona humana $»^{25}$. Esta última categoría pone además de manifiesto que repetición y gravedad no van necesariamente de la mano.

Por otro lado, la práctica muestra que el procedimiento de sentencia piloto se ha aplicado en un número limitado de situaciones (Buyse, 2016: 109), que no siempre puede considerarse que son generadoras de "graves violaciones de los derechos humanos», aunque pueda tratarse de deficiencias "que ponen gravemente en peligro la preeminencia del derecho en los Estados correspondientes ${ }^{26}$. Son cuatro las grandes categorías de problemas sistémicos o estructurales que han llevado al TEDH a dictar sentencias piloto en el marco de una jurisprudencia bien consolidada: a) disfunciones legislativas o malas prácticas en materia de derechos patrimoniales ${ }^{27}$; b) duración excesiva del procedimiento $\left.^{28} ; c\right)$ condiciones de detención inhumanas o degradantes ${ }^{29} ; \mathrm{y}$ d) inejecución prolongada de sentencias y de decisiones internas definitivas ${ }^{30}$. En algún procedimiento de sentencia piloto, el TEDH también ha identificado problemas estructurales o sistémicos particulares, que afectan a un único sistema jurídico nacional ${ }^{31}$.

25 TEDH, La politique de priorisation de la Cour, 22 de mayo de 2017.

26 Resolución 1787 (2011) de la Asamblea Parlamentaria, cit., párr. 5.

27 En su sentencia Burmych y otros c. Ucrania (cit., párr. 162), el TEDH cita en esta categoría los asuntos: Hutten-Czapska c. Polonia, Suljagić c. Bosnia-Herzegovina, Maria Atanasiu y otros c. Rumanía, y Ališić et autres [GC].

28 Ejemplos citados son los asuntos: Lukenda c. Eslovenia, Rumpfc. Alemania, Vassilios Athanasiou y otros c. Grecia, Dimitrov et Hamanov c. Bulgarie, Finger c. Bulgaria, Ümmühan Kaplan c. Turquía, Michelioudakis c. Grecia, Glykantzi c. Grecia, Rutkowski y otros c. Polonia y Gazsó c. Hungría.

29 Se citan los asuntos: Orchowski c. Polonia, Norbert Sikorski c. Polonia, Ananyev et autres c. Russie, Torreggiani y otros c. Italia, Neshkov y otros c. Bulgaria, Varga y otros c. Hungría, y Rezmiveş y otros c. Rumanía.

30 El TEDH cita los asuntos: Bourdov c. Russie ( $n^{\circ}$ 2), Olaru et autres c. Moldova, Youri Nikolayevich Ivanov c. Ukraine, Manushaqe Puto et autres c. Albanie, y Gerasimov et autres $c$. Russie.

31 Résolution 1914 (2013) de la Assemblée parlementaire, Assurer la viabilité de la Cour de Strasbourg: les insuffisances estructurelles dans les Etats Parties, 22 janvier 2013, párr. 3. En la sentencia Burmych y otros c. Ucrania (cit., párr. 162), el TEDH menciona una 
En el ámbito de la Unión Europea, una noción similar, las «deficiencias sistémicas», ha sido utilizada por el Tribunal de Justicia de la Unión Europea (TJUE) vinculada con el respeto de los derechos humanos — primero en materia de asilo ${ }^{32}$ y después en el marco de la Orden de Detención Europea $(\mathrm{ODE})^{33}$ - , pero también por la Comisión Europea, en este caso en cambio para definir posibles amenazas al Estado de derecho ${ }^{34}$. Este concepto jurídico

quinta categoría «cajón desastre», en la que estarían incluidos asuntos relacionados con la prohibición de votar para los detenidos condenados (Greens y M.T. c. Reino Unido) o con la pérdida del estatuto de residente permanente tras una sucesión de Estados (Kuric y otros c. Eslovenia), pero también otros (Xenides-Arestis c. Turquía, Kauczor c. Polonia, M.C. y otros y W.D. c. Bélgica).

32 Sentencia del TJUE (Gran Sala) de 21 de diciembre de 2011, N.S. y otros (C-411/10 y C-493/10), ECLI:EU:C:2011:865. En este asunto, el TJUE se refirió a las «deficiencias sistémicas» (párr. 86), contraponiéndolas a las situaciones de vulneración menores (párrs. 84 y 85). En este sentido, véase también Von Bogdandy y Ioannidis (2014: 59). El TJUE invoca como inspiración la jurisprudencia del TEDH, en particular el caso M.S.S. (TEDH, M. S. S. c. Belgique et Grèce [GC], no 30696/09, Sentencia de 21 de enero de 2011), citando expresamente los párrs. 358, 360 y 367 de este asunto (N. S. y otros, párr. 88).

33 Sentencia del TJUE (Gran Sala) de 5 de abril de 2016, Aranyosi y Căldăraru (C404/15 y C-659/15/PPU), ECLI:EU:C:2016:198. En este asunto, el TJUE mencionó la existencia de «deficiencias sistémicas o generalizadas que afecten a ciertos grupos de personas o a ciertos centros de reclusión» (párr. 89), que vinculó a una violación de la prohibición de tratos inhumanos o degradantes. Véase también la Sentencia del TJUE (Gran Sala) de 25 de julio de 2018, Minister for Justice and Equality (C-216/18 PPU), ECLI:EU:C:2018:586, párrs. 34, 60, 61, 68 y 74. En ella el TJUE también alude a "deficiencias sistémicas o generalizadas», esta vez en relación con la independencia del poder judicial de un Estado miembro, constitutivas de una violación del contenido esencial del derecho a un proceso equitativo.

34 Véase, por ejemplo, Comisión Europea, Comunicación de la Comisión al Parlamento Europeo y al Consejo, Un nuevo Marco de la UE para reforzar el Estado de Derecho, COM (2014) 158 final, 11 de marzo de 2014. El principal objetivo de este Marco es permitir a la Comisión «hacer frente a las amenazas que pesan sobre el Estado de Derecho que tengan un carácter sistémico" presentes en un Estado miembro, a través de un diálogo entre ambos con el fin de evitar la escalada de la amenaza sistémica y "que pueda convertirse en un "riesgo claro de violación grave", lo que podría activar el procedimiento del art. 7 del TUE», esto es, una reacción más contundente de la UE. El Marco se activaría, por tanto, «en situaciones en las que las autoridades de un Estado miembro estén adoptando medidas o tolerando situaciones que pueda afectar sistemática y negativamente a la integridad, la estabilidad o el buen funcionamiento de las instituciones y los mecanismos de salvaguardia establecidos a nivel nacional 
desarrollado para referirse a insuficiencias internas de carácter grave no se corresponde, por tanto, con el término "problema sistémico» utilizado por el TEDH (y con el mismo sentido por el Comité de Ministros y por la Asamblea Parlamentaria $\left.{ }^{35}\right)$, aunque se haya querido encontrar para aquel concepto un apoyo adicional en el derecho del Consejo de Europa (por ejemplo, por Von Bogdandy y Ioannidis, 2014: 30). Cuestión distinta es si estas «deficiencias sistémicas» serían en todos los supuestos constitutivas de violaciones graves, sistemáticas (que no sistémicas) o generalizadas de los derechos humanos en el sentido que tienen estos términos en el Derecho Internacional de los Derechos Humanos. Pero este interrogante excede nuestro objeto de estudio.

En cualquier caso, esta digresión no debe hacernos perder de vista dos datos esenciales para nuestro discurso principal. En primer lugar, no todo problema sistémico o estructural da lugar a un procedimiento de sentencia piloto. Sin embargo, no existe un criterio de fondo inequívoco que permita anticipar, en todos los casos, la decisión del TEDH al respecto (Queralt Jiménez, 2018: 408). En segundo lugar, tampoco se conocen los elementos que llevan al TEDH a preferir una determinada demanda respecto del resto de demandas similares y tramitarla como asunto piloto, al no hacerlo sobre la base de criterios claramente definidos (Glas, 2016: 49; Di Marco, 2016: 905; Leach et al., 2010b: 34; Queralt Jiménez, 2018: 408). Sin embargo, la decisión de iniciar un procedimiento de sentencia piloto y la elección del demandante piloto frente al resto de demandantes clones supone su sometimiento a una tramitación diferente, cuyo alcance e impacto en el sistema del CEDH es una de las cuestiones que pretendemos evaluar en el presente trabajo.

\section{LA SENTENCIA PILOTO}

Aunque inicialmente las sentencias piloto no respondieron siempre a un mismo formato, el art. 61 del Reglamento exige («deberá») al TEDH que incorpore en su sentencia piloto un contenido mínimo obligatorio, consistente en «indicar»: a) el problema estructural o sistémico o disfunción que haya

para garantizar el Estado de Derecho». Véanse COM(2014) 158 final, punto 4.1; Recomendación (UE) 2016/1374 de la Comisión, de 27 de julio de 2016, relativa al Estado de derecho en Polonia, DOUE L 217 de 12.8.2016, p. 53, en particular párrs. 4 y 6 Preámbulo; Comisión Europea, Propuesta motivada de conformidad con el art. 7, apdo. 1, del TUE por lo que respecta al Estado de derecho en Polonia, Propuesta de decisión del Consejo relativa a la constatación de un riesgo claro de violación grave del Estado de Derecho por parte de la República de Polonia, COM(2017) 835 final, de 20 de diciembre de 2017, párr. 7 [cursivas añadidas].

35 Véanse las notas 6 y 21. 
constatado, b) el tipo de medidas correctoras que el Estado demandado debe adoptar a nivel interno (art. 61.3).

En la práctica esta disposición se ha traducido en que, en la parte argumentativa de la sentencia piloto, tras pronunciarse, como en cualquier otra sentencia, sobre si ha habido alguna violación material del Convenio en el caso particular examinado, se abre un epígrafe dedicado al art. 46 del Convenio que cuenta al menos con dos apartados: uno en el que justifica la aplicación del procedimiento de sentencia piloto y otro en el que se refiere a las medidas correctoras necesarias.

En particular, en la parte argumentativa el tribunal da «indicaciones» al Estado sobre las medidas correctoras que debe adoptar ${ }^{36}$. En algunos casos, el tribunal ha proporcionado indicaciones de ejecución detalladas ${ }^{37}$, en otros ha indicado una serie de opciones para resolver el problema estructural identifi$\mathrm{cado}^{38}$, mientras que en un tercer grupo sus indicaciones han sido más vagas ${ }^{39}$. La práctica del tribunal ofrece, por tanto, un amplio abanico de aproximaciones a esta cuestión, lo que se ha dicho obedece a «la flexibilidad necesaria para atender la gama de diferentes situaciones a las que se enfrenta» (Wildhaber, 2009: 74). Para algunos, el tribunal debiera añadir que las medidas que indica no son exclusivas para evitar que el Estado pudiera llegar a objetar al Comité de Ministros que una medida general de ejecución que le recomienda no figuraba en la sentencia piloto y, por tanto, no debe adoptarla (Szymczak, 2011: 285).

En cambio, en la parte operativa o fallo de la sentencia piloto, y más allá de su práctica inicial (por ejemplo, en los asuntos Broniowski y Hutten Czaps$k a$ [GS] c. Polonia) y de algún otro ejemplo aislado (como W.D. c. Bélgica), el tribunal no suele identificar la naturaleza del problema (Glas, 2016: 58). Por el contrario, sí es habitual la mención en el fallo del tipo de medidas correctoras

36 Aunque en algunos casos, en efecto, por su propia naturaleza, los términos empleados son incondicionales y dejan poco margen de apreciación a los Estados (como Xenides-Arestis c. Turquía), en la mayoría se considera preferible hablar de «indicaciones» (Glas, 2016: 52-55; Szymczak, 2011: 280 y 281).

37 Por ejemplo, en Manushaque Puto y otro c. Albania, cit., párrs. 110-118.

38 Por ejemplo, en Maria Atanasiu y otros c. Rumanía, párrs. 229-236; Gerasimov y otros c. Rusia, cit., párr. 224.

39 Por ejemplo, en Greens y M.T. c. Reino Unido, cit., párr. 114. La falta de indicaciones detalladas suele coincidir con violaciones que derivan de la superpoblación carcelaria o de la dilación en los procesos (Di Marco, 2016: 901 y 902). Podría incluirse en este grupo la sentencia piloto Hutten-Czapska c. Polonia, ya que el tribunal se limita a reenviar las recomendaciones dadas por el Tribunal Constitucional polaco (cit., párr. 239), a pesar de que ello en la práctica suponga un mayor detalle. 
que deben ser adoptadas por el Estado, en sintonía con lo que dispone el art. 61.6.a ${ }^{40}$. La formulación que adopta esta mención en el fallo es imperativa («must») (Haider, 2013: 45), aunque redactada en términos muy generales. Básicamente, el tribunal se limita a identificar si las medidas correctoras deben ir dirigidas a resolver la disfunción crónica identificada (preventivas), a reparar los daños sufridos causados por el problema (reparadoras) o si puede ser necesaria una combinación de ambas ${ }^{41}$, dejando de este modo un cierto margen de actuación al Estado, incluso en los casos en que le ha dado indicaciones detalladas en la parte argumentativa ${ }^{42}$.

Además de este contenido mínimo obligatorio, el art. 61 del Reglamento permite que el tribunal incorpore en su sentencia otro tipo de contenidos, que tendrán, por tanto, carácter opcional. En primer lugar, el tribunal puede reservarse, en todo o en parte, la cuestión de la satisfacción equitativa a la espera de que se adopten las medidas indicadas (art. 61.3 y 5). El TEDH ha hecho uso de esta posibilidad en algunas ocasiones (como en Broniowski c. Polonia), mientras que en otras en la misma sentencia piloto ha resuelto la cuestión de la satisfacción equitativa (como en Rutkowski y otros c. Polonia y Rezmives y otros c. Rumanía).

En segundo lugar, el TEDH «puede» fijar, en el fallo de la sentencia piloto, un plazo determinado para la adopción de estas medidas, «tomando en cuenta la naturaleza de las medidas requeridas y la rapidez con la que se puede remediar, a nivel interno, el problema que hubiera constatado» (art. 61.4). En la práctica, salvo en los asuntos Broniowski (párr. 198) y Hutten-Czapska [GS] (párr. 247) c. Polonia, en donde se limitó a hablar de un plazo razonable en la parte argumentativa de la sentencia piloto (Glas, 2016: 56 y 59), el tribunal siempre ha hecho uso de esta posibilidad. Si bien el plazo más habitual ha sido

40 El art. 61.3 se refiere únicamente a la «sentencia», por contraposición, por ejemplo, al párr. 4 o al párr. 6.a), que sí usan los términos "fallo de la sentencia»; diferencia terminológica que sustentaría la práctica seguida por el TEDH.

41 Así, por ejemplo, en los casos vinculados con la no ejecución de decisiones judiciales nacionales y con la ausencia de recursos internos, así como con la lentitud en los procedimientos judiciales, el TEDH suele focalizar las medidas correctoras en las medidas reparadoras (asuntos Burdov c. Rusia (no 2), cit., párr. 6 dictum; Olaru y otros c. Polonia, cit., párr. 5 dictum; Rumpfc. Alemania, cit., párr. 5 dictum), mientras que en asuntos como Hutten-Czapska c. Polonia (cit., párr. 4 dictum), María Atanasiu y otros c. Rumanía (cit., párr. 6 dictum) o Greens y MT c. Reino Unido (cit., párr. 6 dictum) indica medidas preventivas, y en otros, como Ananyev y otros $c$. Rusia (cit., párrs. 7 y 8 dictum) o Torreggiani y otros c. Italia (cit., párr. 4 dictum), clarifica que son necesarios ambos tipos de medidas (Glas, 2016: 52).

42 Véase, por ejemplo, Manushaque Puto y otros c. Albania, cit., párr. 6 dictum. 
de un año a contar desde la fecha en que la sentencia piloto sea definitiva en virtud del art. 44.2 $\mathrm{CEDH}^{43}$, también ha fijado plazos de seis meses ${ }^{44}$, de 18 meses $^{45}$ e incluso de dos años ${ }^{46}$.

En tercer lugar, el tribunal también puede «aplazar el examen de demandas fundadas en la misma alegación hasta que se adopten las medidas correctoras indicadas en el fallo de la sentencia piloto" (art. 61.6.a). El aplazamiento no es, por tanto, una consecuencia automática de la sentencia piloto. Esto parece lógico si se tiene en cuenta que esta medida no parece apropiada «en los casos en los que la seguridad o el bienestar de la persona están en riesgo» (Leach et al., 2010b: 30). Sin embargo, la naturaleza de los derechos violados no siempre ha guiado la decisión del tribunal en su decisión relativa al aplazamiento ${ }^{47}$.

En la práctica, el aplazamiento ha sido desigualmente aplicado, generando diferentes tipos de situaciones (Glas, 2016: 57). Mientras en unos casos el tribunal ha continuado con la tramitación de todas las demandas similares («por el procedimiento normal») ${ }^{48}$, en otros ha aplazado el examen de todas ellas (aunque no necesariamente sometiéndolas a un mismo tratamiento) ${ }^{49}$,

43 Véase el fallo de Alisic y otros c. Bosnia-Herzegovina, Croacia, Serbia, Slovenia y la ex-República yugoslava de Macedonia [GC]; Rumpf c. Alemania; Athanasoiu y otros c. Grecia; Dimitrov y Hamanov c. Bulgaria; Finger; Ümmühan Kaplan c. Turquia; Michelioudakis c. Grecia; Glykantzi c. Grecia; Gazso c. Hungria; Ivanov c. Rusia; Torreggiani y otros c. Italia; Kuric y otros c. Eslovenia.

44 Véase el fallo de Suljagic c. Bosnia-Herzegovina; M.C. y otro c. Italia; Burdov c. Rusia (no 2); Greens y M.T. c. Reino Unido; Rezmives y otros c. Rumania; Ananyev y otros.

45 Véase el fallo de María Atanasiu y otros c. Rumanía; Manushage Puto y otros c. Albania; Neshkov y otros c. Bulgaria.

46 Véase el fallo de W.D. c. Bélgica.

47 Ejemplos de aplazamiento en casos en que el problema sistémico afectaba a los derechos más fundamentales del art. 3 son Ananyev y otros c. Rusia, Neshkov y otros c. Bulgaria o Varga y otros $c$. Hungría, ad exemplum; mientras que no se adoptó esta decisión en otros supuestos que también afectaban al art. 3 (como Torreggiani y otros $c$. Italia o Greens y M.T. c. Reino Unido), lo que sugiere que concurren otros criterios además de la naturaleza de los derechos violados para esta decisión (Di Marco, 2016: 904-906). El TEDH justifica esta decisión por la naturaleza de los derechos en juego y la importancia y la urgencia de las demandas. Por ejemplo, en los asuntos Rumpf c. Alemania (cit., párr. 75), Vassilios Athanasiou y otros c. Grecia (cit., párr. 58), Finger c. Bulgaria (cit., párr. 135), Dimitrov y Hamanov c. Bulgaria (cit., párr. 133), Ananyev y otros c. Rusia (cit., párr. 236) o Varga y otros c. Hungría (cit., párr. 116).

49 Por ejemplo, la tramitación de todas las demandas ha sido aplazada en los asuntos Broniowski c. Polonia, Hutten-Czapska c. Polonia, Sujjagic, Maria Atanasiu y otros c. Rumanía, Kuric y otros c. Eslovenia, Michelioudakis c. Grecia (cit., párrs. 79-80), 
mientras en un tercer grupo de asuntos ha procedido a una tramitación mixta (examen y aplazamiento) ${ }^{50}$. Este aplazamiento suele fijarse por un tiempo determinado (Glas, 2016: 58), normalmente el tiempo que el Estado demandado necesita para adoptar las medidas requeridas ${ }^{51}$, por lo que ambos plazos suelen ser coincidentes, aunque no tiene por qué ser asi ${ }^{52}$. En todo caso, en la práctica más reciente del TEDH este aplazamiento y las condiciones en que se decide se incorporan al fallo de la sentencia piloto ${ }^{53}$.

Por último, el Reglamento prevé que, una vez dictada la sentencia piloto, el TEDH debe informar de ello a una serie de órganos del Consejo de Europa, como el Comité de Ministros, la Asamblea Parlamentaria, el secretario general y el comisario de Derechos Humanos (art. 61.9) ${ }^{54}$. Se pretende de este modo poner de relieve que las sentencias piloto atañen a todo el sistema europeo de garantía de derechos humanos, ya que requieren, en muchas ocasiones, de la

Glykantzi c. Grecia (cit., párrs. 82-83), mientras que en otros casos se ha establecido un diferente tratamiento en el aplazamiento de las demandas comunicadas y no comunicadas (por ejemplo, en Burdov c. Rusia (no 2), cit., párrs. 144-146; Olaru y otros c. Polonia, cit., párrs. 59-61 y párr. 6 dictum; Gerasimov y otros c. Rusia, cit., párr. 224 y párr. 14 dictum), y en algunos otros se han establecido diferencias en el tratamiento del aplazamiento incluso entre las demandas no comunicadas - ya presentadas y futuras- (Rutkowski y otros c. Rumanía, cit., párrs. 9, 10 y 11 dictum y párr. 227).

50 La distinción inicial era entre demandas presentadas ante el Tribunal antes de la fecha de la sentencia piloto o después (por ejemplo, Puto y otros c. Albania, cit., párrs. 119 a 121). En la actualidad, el TEDH prefiere distinguir entre demandas comunicadas y no comunicadas, para continuar con el examen de las demandas del primer grupo "por el procedimiento normal» y aplazar el examen de las del segundo grupo (por ejemplo, Ümmühan Kaplan c. Turquía, cit., párr. 6 dictum; Torreggiani y otros c. Italia, cit., párr. 5 dictum; Rezmives y otros c. Rumanía, cit., párr. 5 dictum).

51 Por ejemplo, Kurić and Others $v$. Slovenia, no 26828/06, judgment (friendly settlement), 12 de marzo de 2014, párr. 134.

52 Por ejemplo, en el asunto Gerasimov y otros c. Rusia, el TEDH dio a Rusia un plazo de un año para la adopción de las medidas generales (cit., párrs. 223-226 y párr. 12 dictum), mientras que fijó un aplazamiento de dos años (cit., párr. 229 y párr. 14 dictum).

53 Por ejemplo, véase el fallo de las sentencias Rezmives y otros $c$. Rumanía y Rutkowski y otros c. Polonia. Pero esto no siempre fue así. Por ejemplo, en el asunto Broniowski c. Polonia, la decisión de aplazamiento fue posterior a la sentencia piloto (TEDH, Communiqué du Greffier 400, Ajournement des affaires concernant les "biens situés au-delà du Boug», 31.8.2004).

$54 \mathrm{El}$ art. 61.10 añade que todas las decisiones y sentencias adoptadas en el marco de un procedimiento de sentencia piloto se darán a conocer a través del sitio web del TEDH. 
concurrencia de diferentes actores para la reversión de la disfunción crónica (Queralt Jiménez, 2018: 409).

\section{LA EJECUCIÓN DE LA SENTENCIA PILOTO}

Aunque la ejecución de la sentencia piloto no suele ser sencilla y requiere tiempo (a veces décadas, como el asunto Ivanov c. Ucrania), el art. 61 del Reglamento, sin embargo, únicamente incorpora cuatro disposiciones al respecto. En primer lugar, como vimos, prevé que durante esta fase el tribunal puede pronunciarse sobre la satisfacción equitativa si se hubiere reservado esta cuestión (art. 61.5). Esta sentencia separada de la sentencia sobre el fondo, que pone fin al procedimiento respecto del demandante piloto, pero no al procedimiento piloto en cuanto tal, incorpora un epígrafe dedicado al art. 46 $\mathrm{CEDH}$ en el que el TEDH entra a evaluar la puesta en práctica por el Estado condenado de las medidas generales necesarias ${ }^{55}$.

En segundo lugar, el Reglamento dispone que: «Si las partes, en un asunto piloto, alcanzan un acuerdo amistoso, este deberá contener una declaración de la Parte contratante demandada relativa a la puesta en práctica de las medidas generales indicadas en la sentencia y las medidas correctoras que deban ser establecidas para los demás demandantes, declarados o potenciales» (art. 61.7). Esta disposición, haciéndose eco de la práctica existente hasta el momento de su adopción ${ }^{56}$, contempla únicamente los acuerdos amistosos a celebrar con el demandante piloto, si bien es cierto que en este marco el TEDH no solo ha entrado a valorar la situación particular de este demandante, sino también las medidas que pretenden resolver la deficiencia general subyacente, aunque le ha bastado una promesa firme por parte del Estado de que se van a tomar tales medidas ${ }^{57}$. A pesar del tenor literal de esta disposición, la práctica posterior muestra ejemplos de acuerdos amistosos con los demandantes clones $^{58}$, pero también de declaraciones unilaterales ${ }^{59}$, en consonancia con el

55 Por ejemplo, Kurić and others $v$. Slovenia (application $n^{\circ} .26828 / 06$ ), judgment (just satisfaction), 12 de marzo de 2014, párrs. 131 y ss.

56 Broniowski c. Poland [GC], arrêt (règlement amiable), 28.9.2005; Hutten-Czapska c. Poland [GC], arrêt (règlement amiable), 28.4.2008.

57 Broniowski c. Pologne (règlement amiable), cit., párrs. 36, 37 y 42; Hutten-Czapska c. Pologne (règlement amiable), cit., párrs. 34, 25 y 43; Kurić and Others v. Slovenia (friendly settlement), cit., párr. 135.

58 Por ejemplo, asuntos Ivanov c. Rusia o Rutkowski y otros c. Polonia.

59 Así, por ejemplo, en cuanto a la ejecución de la sentencia piloto en el asunto Rutkowki y otros c. Polonia, véase Załuska v. Poland (application n ${ }^{\circ}$. 53491/10) and Rogalska v. Poland (nº 72286/10) and 398 other applications, decisión, 22 June 2017, párrs. 11-13 
compromiso asumido en el proceso de reforma del sistema del CEDH de reforzar este tipo de vías de actuación para aligerar la carga del tribunal y para llegar a una solución rápida y satisfactoria para todas las partes ${ }^{60}$. Cuando el TEDH acepta estos acuerdos amistosos y declaraciones unilaterales, se archivan las demandas afectadas, ya que se les ha ofrecido una solución ad hoc por la vía no contenciosa en el marco del proceso de ejecución de las medidas generales requeridas ${ }^{61}$, lo que no supone, sin embargo, por sí mismo la terminación formal del procedimiento de sentencia piloto ${ }^{62}$.

Por último, el Reglamento establece que el TEDH «podrá en cualquier momento examinar una demanda aplazada si así lo exige el interés de una buena administración de la justicia» (art. 61.6.c) y que, si el Estado demandado «no acatara el fallo de la sentencia piloto, el TEDH, salvo decisión en contrario, retomará el examen de las demandas aplazadas» (art. 61.8). Entendemos que la primera disposición se refiere a un supuesto de revocación de la decisión de aplazamiento cuando el plazo inicialmente previsto todavía no ha concluido, mientras que en la segunda el aplazamiento ha terminado y que, dado que el Estado demandado «deja sin resolver el problema y continúa violando el Convenio", el TEDH debe reanudar el examen de las demandas clones. En la práctica, esta decisión de reanudación se ha adoptado formalmente únicamente en casos extremos de no acatamiento ${ }^{63}$.

y 53-55; y ECtHR, 400 applications against Poland struck out, Press Release no 217 (2017), 22.06.2017.

60 Comité des Ministres, Résolution Res(2002)59 relative à la pratique en matière de règlements amiables, 18 décembre 2002; Déclaration d'Interlaken, Plan d'action, párr. D.7.a).i); TEDH, Rapport annuel 2011, p. 8; nuevo art. 62A Reglamento TEDH («Declaraciones unilaterales»).

61 Comité des Ministres, H46-35 Yuriy Nikolayevich Ivanov, groupe Zhovner et Burmych et autres $c$. Ukraine (requête $\mathrm{n}^{\circ}$ 40450/04 et autres), Surveillance de l'exécution des arrêts de la Cour européenne, 1348 réunion, 4-6 juin 2019 (DH), Doc. CM/Del/ Dec(2019)1348/H46-35, 6 de junio de 2019, párrs. 6, 7, 8 y 9.

62 Por ejemplo, en el asunto Rutwoski, aunque el TEDH decidió archivar unas 400 demandas tras diferentes acuerdos amistosos y declaraciones unilaterales, no ha tomado la decisión formal de dar por terminado el procedimiento de sentencia piloto, ni siquiera cuando el Comité de Ministros decidió dar por terminado su examen de 103 de estos casos (Committee of Ministers, Resolution CM/ResDH(2017)426, Execution of the judgments of the European Court of Human Rights 103 cases against Poland, 7 de diciembre de 2017).

63 Véase, por ejemplo, ECtHR Registrar, Court Decides to Resume Examination of Applications concerning Non-enforcement of Domestic Decisions in Ukraine, Press Release, 29 de febrero de 2012; Kharuk and Others v. Ukraine (app. no 703/05 and others), 
La práctica posterior muestra otro tipo de desarrollos en la fase de ejecución no previstos en el Reglamento. Así, por ejemplo, en ocasiones el TEDH, normalmente a petición del Estado demandado, y después de valorar las dificultades que este enfrenta a la hora de acatar el fallo (por ejemplo, por la insuficiencia del tiempo dado para la adopción de las medidas correctoras necesarias), ha decidido en algunos casos la prórroga de los plazos fijados ${ }^{64}$. Esta prórroga implica la modificación a posteriori de un elemento del fallo de su sentencia piloto, lo que podría afectar negativamente la autoridad del TEDH, por lo que parece recomendable que el TEDH intente fijar en el fallo plazos lo más realistas posibles (Wallace, 2011: 5 y 6; Leach et al., 2010a: 358; Glas, 2016: 66).

Por otro lado, en los casos en que no acordó el aplazamiento de las demandas similares, o cuando este ha terminado, el TEDH continúa su tramitación a través del procedimiento normal y, en ausencia de acuerdo amistoso o declaración unilateral, dicta una sentencia sobre el fondo del asunto examinado (de manera individual o conjuntamente una vez adoptada la decisión de unir las demandas), aprovechando la ocasión para testar y pronunciarse sobre la efectividad de las medidas generales adoptadas por el Estado.

El Estado correspondiente puede, por tanto, enfrentarse a sucesivas nuevas condenas por parte del TEDH, como ha ocurrido, en particular, en el marco de los cuatro procedimientos de sentencia piloto que han contado con Estados con una actitud más reticente, recalcitrante o desafiante (Burdov c. Rusia (no 2), Gerasimov y otros c. Rusia, Ivanov c. Ucrania y Greens y M.T. c. Reino Unido $)^{65}$. En estas condiciones, el TEDH entiende que sigue siendo el

judgment, 26 de julio de 2012, párr. 7. Glas entiende que esta decisión también se ha adoptado en el asunto Greens y M.T. c. Reino Unido (2016: 63), el 24 de septiembre de 2013, al decidir en el marco de este procedimiento retomar el examen de las más de 2000 demandas similares aplazadas (veáse también TEDH, Fiche thématique - Les arrêts pilotes, cit., 12).

64 Véanse, por ejemplo, Maria Atanasiu y otros c. Rumanía (dos prórrogas); Olaru y otros c. Polonia (dos prórrogas); Greens y M.T. c. Reino Unido (dos prórrogas). Sin embargo, en el asunto Kurić and Others, si bien el tribunal prorrogó el plazo respecto de la satisfacción equitativa, rechazó las dos peticiones de prórroga para el establecimiento de medidas correctoras a nivel nacional planteadas por el Estado (Kuric and Others v. Slovenia (friendly settlement), cit., párr. 11). TEDH, Fiche thématique-Les arrêts pilotes, cit.

65 Véase, por ejemplo, en el marco del procedimiento Burdov c. Rusia (no 2): Ilyushkin and Others v. Russia, $\mathrm{n}^{\circ}$ 5734/08 and 28 others, Judgment (Merits and Just Satisfaction), 17.04.2012 y Kalinkin and Others, $\mathrm{n}^{\circ} 16967 / 10$ and 20 others, Judgment (Merits and Just Satisfaction), 17.04.2012; en el marco del procedimiento Gerasi- 
único medio para los demandantes de defender efectivamente su derecho y obtener una reparación ${ }^{66}$, que se ve compelido «a pronunciarse sobre tales demandas como un tribunal de primera instancia ${ }^{67}$, pero no cabe duda de que esta no es la solución al problema. Resulta fundamental un cambio de actitud por parte de los Estados.

Para forzar este cambio, el TEDH en alguna ocasión ha recurrido al procedimiento simplificado y acelerado ante un Comité de tres jueces con el fin de agilizar la condena. Para ello ha considerado que se trataba de un asunto repetitivo ligado a una jurisprudencia consolidada del tribunal en los términos previstos en el art. $28 \mathrm{CEDH}$ tras la reforma del Protocolo no 14 (Di Marco, 2016: 912 y 913; Kindt, 2018: 176 y 177) ${ }^{68}$. La continua resistencia de Ucrania en el procedimiento Ivanov c. Ucrania ha urgido un cambio de enfoque por parte del tribunal, dando lugar a nuevos desarrollos en este ámbito.

El primero fue la utilización de un procedimiento abreviado, simplificado y acelerado denominado «fast-track procedure». No se trata de una mera acumulación de dos o más demandas (art. 42 Reglamento) ni del procedimiento abreviado ante el Comité de tres jueces (art. $28 \mathrm{CEDH})$, sino

mov y otros c. Rusia: Shtolts and Others $v$. Russia, no 77056/14 and others, decision (admissibility), 22.02.2018; en el marco del procedimiento Greens y M.T. c. Reino Unido: Registrar Court, Court finds violation of the right to vote in ten follow-up prisoner voting cases but awards no compensation or legal costs, Press Release no 239 (2014), 12.08.2014; Remaining legacy prisoner voting cases: ECHR finds violation of the right to vote but awards no compensation or legal costs, Press Release no ECHR 052 (2015), 10.02.2015; McLean and Cole v. the United Kingdom, $\mathrm{n}^{\text {os }}$. 12626/13 and 2522/12, decision, 11.06.2013; Firth and Others $v$. the United Kingdom, $\mathrm{n}^{\circ} .47784 / 09$ and nine others, 12.08.2014; McHugh and Others v. the United Kingdom, no. 51987/08 and 1,014 others, judgment (committee), 10.02.2015; Millbank et autres c. Royaume-Uni, no 44473/14 and 21 others, sentencia de 30 de junio de 2016; Miller et autres $c$. Royaume-Uni, no 70571/14 and 6 others, sentencia (comité) de 11 de abril de 2019; TEDH, Fiche thématique - Droit de vote des détenus, avril 2019; y en el marco del procedimiento Ivanov c. Ucrania: Kharuk and Others v. Ukraine, no 703/05 and 115 others, Judgment (Merits and Just Satisfaction), 26.07.2012; Rozhenko and Others $v$. Ukraine, no 2644/04 and 23 others, sentencia (committee), 11.04.2013; Kononova and Others $v$. Ukraine, $\mathrm{n}^{\circ} 11770 / 03$ and 89 other applications, sentencia (Merits and Just Satisfaction), 06.06.2013; Burmych et autres c. Ukraine [GC], cit.

66 Véanse, por ejemplo, Ilyushkin and Others, cit., párrs. 43 y 75; Kalinkin and Others, cit., párrs. 37 y 60 .

67 Gerasimov y otros c. Rusia, cit., párr. 206.

68 Véanse, por ejemplo, McHugh and Others v. the United Kingdom; Miller et autres $c$. Royaume-Uni; Rozhenko and Others v. Ukraine. 
de un procedimiento que permite adoptar de manera agrupada sentencias y decisiones de archivo, cuyo contenido queda esencialmente limitado al reconocimiento de la violación y a la concesión de una satisfacción equitativa conforme a unos criterios estándar, lo que permite que los demandantes obtengan rápidamente una reparación económica ${ }^{69}$.

La persistencia del problema decidió al TEDH a dar un paso más. En su sentencia de 12 de octubre de 2017 en el asunto Burmych y otros c. Ucrania, entendió que no servía a ningún propósito que siguiera examinando estos casos y que su solución no era competencia del tribunal, puesto que las cuestiones jurídicas estaban resueltas, sino que, dado que las dificultades a enfrentar eran de orden político y financiero, incumbía al Estado y al Comité de Ministros asumir sus responsabilidades ${ }^{70}$. En consecuencia, sin haber oído a los demandantes, archivó directamente las demandas y se las envió al Comité de Ministros, órgano político del Consejo de Europa del que forma parte el Estado incumplidor, con el fin de que fueran tratadas en el marco de las medidas generales de ejecución de la sentencia piloto Ivanov c. Ucrania ${ }^{71}$, advirtiendo que quedarían sometidas al mismo tratamiento las demandas similares futuras $^{72}$. Esto no supuso, sin embargo, el fin del procedimiento piloto, ya que el TEDH se reservó la facultad de reinscribir en el registro las demandas del caso Burmych y otros c. Ucrania y "toda otra demanda similar futura" si las circunstancias lo justifican (art. 37.2 CEDH), previendo incluso un plazo de dos años al término del que reexaminaría la situación para verificar si concurrían estas circunstancias ${ }^{73}$. Por el momento, el Comité de Ministros se ha limitado a seguir lidiando con la implementación de la sentencia Ivanov c. Ucrania ${ }^{74}$, lo que puede verse como un consentimiento tácito a este enfoque (Ulfstein y Zimmermann, 2018: 18) o como un posicionamiento puntual frente a una situación excepcional.

Esta forma de proceder no ha tenido, sin embargo, continuidad. En 2018, durante el examen de un nuevo asunto en el marco del procedimiento Gerasimov y otros c. Rusia, el TEDH entendió que la situación era visiblemente diferente al asunto Burmych y otros c. Ucrania, por lo que decidió continuar

69 Kharuk and Others (116 demandantes); Kononova and Others (90 demandantes). Véase este procedimiento también en Burmych et autres c. Ukraine, cit., párrs. 28, 29, 43, $147,151,170$.

70 Burmych et autres c. Ukraine, cit., párrs. 195, 196, 197 y 199.

71 Ibid., dictum, punto 4.

72 Ibid., párrs. 221 y 222.

73 Ibid., párr. 223.

$74 \mathrm{CM} / \mathrm{ResDH}(2019) 153$, Exécution des arrêts de la Cour européenne des droits de l'homme, 352 affaires contre Ukraine, 6 juin 2019. 
con el examen de las demandas individuales en el modo habitual ${ }^{75}$. Pero, al no realizar ninguna apreciación adicional al respecto, deja la puerta abierta a la posible réplica en el futuro de una solución que, como veremos, genera importantes retos jurídicos y políticos para el conjunto del sistema del CEDH y que, por lo demás, no ha resuelto el caso Ivanov c. Ucrania.

No obstante, si bien existen supuestos especialmente sangrantes de incumplimiento, la valoración que el tribunal está haciendo de las medidas adoptadas por los Estados en ejecución de sentencias piloto está resultando en la mayoría de los casos positiva ${ }^{76}$, lo que, dependiendo del tipo de medidas generales adoptadas, le lleva a tomar una de estas dos decisiones. O bien, en el caso de que estas medidas incluyan una nueva vía de derecho - indemnizatorio o de otro tipo- - el tribunal declara inadmisibles las demandas similares objeto del litigio por no agotamiento de las vías de recurso internas ${ }^{77}$ y esto de

75 Shtolts and Other v. Russia (admissibility), cit., párr. 95.

76 A la hora de hacer esta valoración, al tribunal le ha bastado en ocasiones que tales medidas generales ofrezcan posibilidades razonables de proporcionar una compensación al demandante, aunque la medida lleve poco tiempo en vigor o no puedan apreciarse sus resultados a largo plazo (Association of Real Property Owners in Eódź and Others $v$. Poland, $\mathrm{n}^{\circ} 3485 / 02$ and others, decision, 8 March 2011, párrs. 81 y 88), mientras que en otros supuestos su análisis ha sido más elaborado (Balakchiev and Others v. Bulgary, $\mathrm{n}^{\circ}$ 65187/10, decision (admissibility), 18 June 2013, párr. 58). Véanse al respecto Glas, 2016: 63-65; Kindt, 2018: 176 y 177.

77 Burmych et autres $c$. Ukraine, cit., párr. 163. Son ejemplos de estas decisiones de inadmisibilidad: Demopoulos and Others v. Turkey [GC], $\mathrm{n}^{\text {os }} .46113 / 99$ and others, decision (admissibility), 1 de marzo de 2010 (en el marco del procedimiento Xenides-Arestis c. Turquía); Nagovitsyn and Nalgiyev v. Russia, $\mathrm{n}^{\text {os }} .27451 / 09$ and 60650/09, decision (admissibility), 23 de septiembre de 2010 (en el marco del procedimiento Burdov v. Russia ( $n^{\circ}$ 2); Eatak v. Poland, $\mathrm{n}^{\circ}$. 52070/08, decision (admissibility), 12 de octubre de 2010 (en el marco de los procedimientos Orchowski c. Polonia y Sikorski); Müdür Turgut and Others v. Turkey, no. 4860/09, decision (admissibility), 26 de marzo de 2013 (en el marco del procedimiento Kaplan c. Turquia); Balakchiev and Others $v$. Bulgaria, no. 65187/10, decision (admissibility), 18 de junio de 2013, y Valcheva and Abrashev v. Bulgaria, $\mathrm{n}^{\text {os }} .6194 / 11$ y 34887/11, decision (admissibility), 18 de junio de 2013 (en el marco de los procedimientos Finger c. Bulgaria y Dimitrov y Hamanov c. Rusia); Techniki Olympiaki A.E. v. Greece, no. 40547/10, decision (admissibility), 1 de octubre de 2013 (en el marco del procedimiento Athanasiou y otros c. Grecia); SteIla and Others $v$. Italy, $\mathrm{n}^{\mathrm{o}}$. 49169/09, decision (admissibility), 16 de septiembre de 2014 (en el marco del procedimiento Torreggiani y otros c. Italia); Xynos v. Greece, $\mathrm{n}^{\circ}$. 30226/09, decision (admissibility), 9 de octubre de 2014 (en el marco de procedimientos Michelioudakis c. Grecia y Glykantzi c. Grecia); Domján v. Hungary, no 5433/17, decision (admissibility), 23 de noviembre de 2017 (en el marco del proce- 
conformidad con el art. 35.1 y 4 CEDH del que deduce la retroactividad del principio de no agotamiento de los recursos internos. Esta decisión, que supone el reenvío a los Estados o la «renacionalización» del asunto, no menoscaba el derecho de los demandantes a presentar una nueva demanda individual si no obtienen una reparación adecuada en el plano nacional ${ }^{78}$. $\mathrm{O}$ bien, cuando los Estados han modificado su legislación para ofrecer a nivel interno una reparación a todas las víctimas poniendo fin a la violación sistémica, el tribunal archiva las demandas similares objeto del litigio, argumentando que este ha sido resuelto en el sentido del art. 37.1.b CEDH, por lo que ya no está justificada la continuación del examen de la demanda, aunque advirtiendo que "podrá decidir que se reinscriba una demanda en el registro cuando estime que las circunstancias lo justifican» (art. 37.2 CEDH) ${ }^{79}$.

Sin embargo, la mera valoración positiva por el tribunal de las medidas correctoras adoptadas no genera por sí misma la terminación del procedimiento de sentencia piloto ${ }^{80}$. Tampoco parece hacerlo el fin de la supervisión por parte del Comité de Ministros ${ }^{81}$. De conformidad con la práctica

dimiento Varga y otros c. Hungría); y Shtolts and Other v. Russia (admissibility), cit. (en el marco del procedimiento Gerasimov y otros c. Rusia).

Burmych et autres c. Ukraine, cit., párr. 164. A modo ejemplificativo, véase Wolkenberg and Others v. Poland, no. 50003/99 and others, decision, 4 de diciembre de 2007, párrs. 72-77, y E.G. v. Poland and 175 Other Bug River applications v. Poland, no 50425/99, decision, ECHR 2008 (extracts), párrs. 22-25 (en el marco del procedimiento Broniowski c. Polonia); Association of Real Property Owners in Eódź and Others v. Poland, no 3485/02 and others, decision, 8 de marzo de 2011, párrs. 82-89 (en el marco del procedimiento Hutten-Czapska c. Polonia); y Anastasov and Others v. Slovenia, no. 65020/13 and others, decision, 18 de octubre de 2016, párrs. 89-102 (en el marco del procedimiento Kuric y otros c. Eslovenia).

80 Así, por ejemplo, en el asunto Broniowski c. Polonia, el TEDH decidió el 4 de diciembre de 2007 archivar los asuntos Wolkenberg et autres c. Pologne, no 50003/99, y Witkowska-Tobola c. Pologne, no 11208/02, seguido en 2008 de un segundo archivo de 110 asuntos y, en octubre, de otros 176 asuntos, momento en que adopta la decisión de finalizar el procedimiento de sentencia piloto (TEDH, La première procédure d'uarrêt pilote» menée à son terme. Fin des "affaires Boug", Communiqué de presse no 691/2008, 6.10.2008).

81 En el procedimiento piloto Kurić y otros c. Eslovenia, la decisión del tribunal de dar por terminado el procedimiento (Anastasov and Others, párrs. 86 y 87) es posterior a la resolución final del Comité de Ministros [Resolution CM/ResDH(2016)112, 25.05.2016]. 
jurisprudencial (Broniowski c. Polonia ${ }^{82}$, Suljagic c. Bosnia-Herzegovina ${ }^{83}$, Hutten-Czapska c. Polonia ${ }^{84}$ y Kuric y otros c. Eslovenia ${ }^{85}$ ), esta terminación parece requerir que el TEDH adopte una decisión formal en la que indique que la disfunción crónica ha sido resuelta y que, por tanto, el tribunal ha cumplido su misión, archivando las demandas análogas y dando por terminado el procedimiento $^{86}$. No obstante, las condiciones materiales necesarias no acaban de quedar claras $^{87}$, lo que genera incertidumbre.

\section{LOS EFECTOS DEL PROCEDIMIENTO DE SENTENCIA PILOTO EN EL SISTEMA DEL CEDH}

La configuración actual del procedimiento de sentencia piloto en los términos que acabamos de identificar deja entrever que en su seno se producen ciertas transformaciones en la relación del TEDH con los demandantes («piloto" y "clones»), pero también en el reparto de competencias con el Estado demandado (vertical) y con el Comité de Ministros (horizontal). Nos interesa ahora determinar el alcance de estos cambios desde esta triple perspectiva con

82 TEDH, La première procédure d'«arrêt pilote» menée à son terme. Fin des "affaires Boug", Communiqué de presse no 691/2008, 6.10.2008.

83 Zadric v. Bosnia and Herzegovina, no. 18804/04, decision, 16.11.2010.

84 TEDH, La Cour européenne des droits de l'homme clôt la procédure d'arrêt pilote dans les affaires polonaises portant sur le "contrôle des loyers» à la suite de l'adoption d'un régime de compensation, Communiqué de presse no 284/2011, 31.03.2011; TEDH, Association des propriétaires fonciers de Eódź et autres c. Pologne, no 3485/02 et autres, decision [Extraits], 08.03.2011.

Anastasov and Others v. Slovenia, $\mathrm{n}^{\circ}$ 65020/13 and others, decision, 18 October 2016. El TEDH advierte, no obstante, que esta decisión se adopta «sans préjudice de toute décision que la Cour pourrait prendre de réinscrire au rôle la présente requête et les autres requêtes suspendues en vertu de l'article $37 \$ 2$ de la Convention ou d'examiner au fond des requêtes introduites ultérieurement si les circonstances le justifient» (Wolkenberg et autres, cit., párr. 77; E.G. c. Pologne et 175 autres affaires de la rivière Boug, cit., párrs. 25, 28 y 29; y Association des propriétaires fonciers de tódź et autres, cit., párr. 90).

El TEDH ha llegado a decidir dar por terminado el procedimiento, a pesar de reconocer que le seguían llegando nuevas demandas análogas cada mes (TEDH, La première procédure d' «arrêt pilote» menée à son terme. Fin des «affaires Boug», Communiqué de presse $n^{\circ}$ 691/2008, 6.10.2008), e incluso, aunque tiempo después de esta decisión, el Comité de Ministros todavía seguía supervisando la ejecución de la sentencia piloto (Glas, 2016: 67). 
el fin de identificar los efectos del procedimiento de sentencia piloto en el sistema del CEDH.

\section{LOS DEMANDANTES «PILOTO»Y LOS DEMANDANTES «CLONES»: EL DERECHO DE RECURSO INDIVIDUAL ANTE EL TEDH}

Como vimos, el procedimiento de sentencia piloto supera los efectos inter partes clásicos, ya que se tramita y resuelve en interés de todas las personas potencialmente afectadas por la misma disfunción crónica subyacente en un orden interno nacional ${ }^{88}$. Pero esta tramitación se hace únicamente sobre la base de un caso particular (el del demandante o demandantes piloto), no integrándose formalmente en el procedimiento al resto de víctimas (los demandantes clones). No se les da ningún tipo de acceso al TEDH (Kindt, 2018: 181 y 182), disponiéndose únicamente que «serán debidamente informados de la decisión de aplazamiento de manera oportuna», y que "[s]e les notificará, si procediera, cualquier nuevo elemento que ataña a su asunto» (art. 61.6.b) Reglamento). No pueden siquiera alegar que el caso piloto no es suficientemente representativo o que su caso es diferente, debiendo ser decidido de manera separada. Al fin y al cabo, la demanda piloto «puede no mostrar los diferentes aspectos que plantea el problema sistémico" ${ }^{89}$ y no todos los demandantes víctimas de una disfunción crónica pertenecen necesariamente a una clase de individuos identificable, sino que pueden encontrarse en situaciones muy diferentes (Leach et al., 2010a: 351). La única posibilidad de ser oídos se produce, sin embargo, en el peor de los escenarios para el TEDH: cuando se reanuda el examen de las demandas similares (Kindt, 2018: 183).

Esta reformulación en el procedimiento de sentencia piloto de la relación entre el TEDH y los demandantes resulta claramente beneficiosa para el tribunal, ya que parece estar contribuyendo al logro del objetivo de reducir su carga de trabajo. Con este procedimiento no solo se anticipa a futuras demandas, sino que el aplazamiento de asuntos similares permite descargar temporalmente al tribunal «de un examen tedioso y sin valor añadido para el interés y calidad de su jurisprudencia» (Ducoulombier, 2011: 268), mientras

88 Broniowski c. Pologne, règlement amiable, cit., párrs. 36, 39; Hutten-Czapska c. Pologne, arrêt au principal, cit., párr. 238; Hutten-Czapska c. Pologne, règlement amiable, cit., párrs. 33, 35, 46, 47; Kuric y otros c. Eslovenia, cit., párr. 90; Wolkenberg and Others, cit., párr. 35; The Association of Real Property Owners in Lodz, cit., párr. 83; Abrisketa Uriarte, 2013: 81; Queralt Jiménez, 2018: 420; Rosu, 2014: 40.

89 Parliamentary Assembly, Resolution 1516 on Implementation of judgments of the European Court of Human Rights, 2 de octubre de 2006, párr. 21; Leach et al., 2010b: 34; Glass, 2016: 49. 
que la renacionalización convierte esta descarga temporal en definitiva (Di Marco, 2016: 903 y 910), si bien nada comparable a la remisión directa al Comité de Ministros para su ejecución de las más de 12000 demandas del caso Burmych y otros c. Ucrania. Los datos de la práctica avalan que este resultado se está consiguiendo ${ }^{90}$, configurando al procedimiento de sentencia piloto como una innovación fundamental en el marco del proceso de reforma para luchar contra la sobrecarga de trabajo del tribunal (Wallace, 2011: 1; Kurban, 2016: 759).

Por lo que al demandante piloto se refiere, la reformulación de esta relación no afecta considerablemente su posición y, en cualquier caso, el saldo es positivo. Se beneficia de un tratamiento individualizado y prioritario de su demanda, que puede conllevar la adopción de medidas individuales, incluida una satisfacción equitativa (Queralt Jiménez, 2018: 422). Además, en los casos en que las medidas generales necesarias consistan únicamente en la adopción de un recurso indemnizatorio en el plano interno, su derecho a ser indemnizado queda adecuadamente reparado por el propio TEDH, incluso en los casos en que se haya reservado esta cuestión para más adelante (Szymczak, 2011: 287).

El tratamiento diferenciado que reciben los demandantes clones también podría resultarles beneficioso al ofrecerles una solución más rápidamente que si se viera su caso individualmente. Algunos elementos, como fijar un plazo para el cumplimiento de la sentencia piloto por el Estado o alentar la celebración de acuerdos amistosos, vendrían a contribuir a este resultado. Además, si tenemos en cuenta que el tribunal suele tardar unos tres años en resolver las demandas individuales, se puede argumentar, por ejemplo, que cuatro años para resolver el problema sistemático detectado en el procedimiento Kuric y otros c. Eslovenia, que afecta a un amplio grupo de personas, es bastante rápido (Kindt, 2018: 184).

Sin embargo, como hemos visto, muchos de los casos no se están resolviendo de un modo tan satisfactorio. A los demandantes clones tal vez les hubiera valido más la pena que el TEDH no hubiera tomado la decisión de

90 Entre 2011 y 2013, coincidiendo con el momento en que el tribunal estaba haciendo un uso mayor del procedimiento de sentencia piloto, el número de demandas pendientes ante él descendió en más de una tercera parte (TEDH, Annual Report 2013, pp. 9 y 26; Sainati, 2015: 156 y 157). También resulta ejemplificativa la inusual fluctuación que se ha producido en la carga de trabajo del tribunal en 2017: el stock de demandas pendientes, que se elevaba a principios de año a 79750, aumentó hasta 93200 en junio y descendió a 56250 a finales de año. Esta diferencia se debió fundamentalmente a la «renacionalización» de demandas en procedimientos de sentencias piloto (TEDH, Analyse statistique 2017, p. 5). 
iniciar un procedimiento de sentencia piloto, continuando el examen de sus demandas caso por caso (Szymczak, 2011: 293). Al fin y al cabo, el aplazamiento del tratamiento de sus demandas supone una vacatio en la protección de sus derechos (Queralt Jiménez, 2018: 422), lo que además implica que su derecho a la reparación conoce un primer desfase respecto del demandante piloto (Szymczak, 2011: 287). Pero incluso en los casos en que no se acuerda el aplazamiento o cuando este ha terminado, su tramitación no es necesariamente prioritaria. Además, en la mayoría de las ocasiones, dado que el tribunal está valorando positivamente las medidas generales adoptadas por los Estados, el examen de estas demandas clones supone su renacionalización. Por otro lado, posiblemente la indemnización que perciban será sensiblemente inferior a la concedida por el TEDH al demandante piloto (Szymczak, 2011: 293). Finalmente, todo ello no impediría que el asunto pudiera volver al TEDH, dilatándose todavía más la duración del proceso, aunque la legítima frustración de los demandantes clones seguramente les desalentará a hacer este último movimiento.

En estas condiciones, cierto es que el procedimiento de sentencia piloto no parece perjudicar la posibilidad de los demandantes clones de plantear pretensiones individuales ante una jurisdicción independiente, imparcial y competente para resolver sobre ellas. Sin embargo, la solución adoptada en el asunto Burmych y otros $c$. Ucrania vendría precisamente a suponer una verdadera denegación de acceso a la justicia internacional, ya que, si bien presenta la ventaja para los demandantes de haber sido colocados automáticamente al mismo nivel que los demandantes Ivanov c. Ucrania, esto mismo representa precisamente la imposibilidad de presentar su caso ante el tribunal, y esto tanto para los demandantes en el asunto Burmych y otros c. Ucrania como para las víctimas futuras, quienes, si no se logra una solución, permanecerán exactamente en la misma posición durante estos dos años (Kindt, 2018: 187). A esto se añade el hecho de que el reenvío al Comité de Ministros supone el inicio de un proceso ante un órgano político, en el que el Estado involucrado queda integrado en la toma de decisiones, que conducirá a una decisión no obligatoria $^{91}$, y en el que no juega el derecho de recurso individual de los demandantes ${ }^{92}$.

91 Burmych et autres c. Ukraine, cit., opinión disidente, párrs. 6 y 13; Kindt, 2018: 187.

92 Règles du Comité des Ministres pour la surveillance de l'exécution des arrêts et des termes des règlements amiables (adoptées par le Comité des Ministres le 10 mai 2006, lors de la 964ème réunion des Délégués des Ministres et amendées le 18 janvier 2017, lors de la 1275 ème réunion des Délégués des Ministres), Règle no 9. 
En cualquier caso, el procedimiento de sentencia piloto sí supone la erosión de los otros aspectos de la dimensión formal del derecho de recurso individual ante el TEDH de los demandantes clones. Menoscaba, sin duda alguna, su derecho a contar con un recurso «efectivo» que satisfaga, por tanto, las garantías del debido proceso, asegurando en su seno el derecho de defensa y una tramitación en un plazo razonable. Pero también resultaría significativamente limitada la dimensión material de este derecho, que requiere que se provea justicia a través de la emisión de una sentencia justa, ajustada a derecho, motivada y ejecutable ${ }^{93}$. Además, el procedimiento de sentencia piloto parece un intento de introducir en el sistema del CEDH "por la puerta de atrás» una suerte de certiorari, al capacitar al TEDH para seleccionar más libremente los casos que quiere examinar (Ulfstein y Zimmermann, 2018: 8-10), cuando este modelo propio del Tribunal Supremo de los Estados Unidos ha sido sistemáticamente rechazado por los Estados a lo largo del proceso de reforma, precisamente porque afectaría al derecho de recurso individual ante el TEDH ${ }^{94}$.

No obstante, se ha considerado que el impacto del procedimiento de sentencia piloto sobre el derecho de recurso individual ante el TEDH no es tan grave, ya que el tribunal vendría por esta vía a proporcionar justicia material a los individuos, si bien indirectamente. Se estaría incrementando su papel "en la provisión del acceso indirecto a la justicia», lo que "también contribuye a la justicia general» (Gerards y Glas, 2017: 18). Este resultado se justifica por la necesidad de incrementar la eficiencia del sistema del Convenio. Se entiende que es mejor limitar el derecho de recurso individual a que el sistema esté totalmente colapsado o desaparezca, razonamiento que resulta particularmente evidente en el asunto Burmych y otros c. Ucrania ${ }^{95}$. Esta tensión presente a lo largo de todo el proceso de reforma parece finalmente haber decantado la balanza hacia una visión más constitucional de la función del TEDH. De

93 Sobre el derecho de acceso a la justicia internacional, véanse, por ejemplo, Acosta Alvarado, 2008: 7, 8 y 14; Gerards y Glass, 2017: 13 y 14; Quispe Remón, 2018: 244; Sardaro, 2005: 45 y 47.

94 Rapport explicatif, Protocole $n^{\circ} 14$..., cit., párr. 34; Report of the Steering Committee for Human Rights (CDDH), The longer-term future of the system of the European Convention on Human Rights, 2016, párr. 91. En un sentido similar, esta propuesta fue rechazada inequívocamente por el Informe del Grupo de Sabios (CM (2006) 203, Report of the Group of Wise Persons to the Committee of Ministers, 15 de noviembre de 2006, párr. 42).

95 Los jueces disidentes afirmaron expresamente que la decisión del tribunal se adoptó en atención a consideraciones de eficiencia y que no tenía nada que ver con los derechos humanos (párr. 1). En el mismo sentido crítico, véase Kindt, 2018: 184. 
hecho, en el marco de este proceso, al mismo tiempo que se consagra el derecho de recurso individual ante el TEDH como piedra angular del sistema ${ }^{96}$, se han ido adoptando otras medidas que reducen su alcance, como la nueva causa de admisibilidad que exige que el demandante haya sufrido un perjuicio importante (art. 35.3.b. CEDH), la obligatoriedad del formulario de demanda (art. 47 Reglamento), la figura del juez único (art. $27 \mathrm{CEDH})$, el recurso a las declaraciones unilaterales (art. 62A Reglamento) o la política de priorización (Gerards y Glas, 2017: 19-29; Lambert Abdelgawad, 2007: 373 y 374).

No obstante, la problemática apuntada revela la necesidad de un cambio de enfoque. Así, por ejemplo, el TEDH podría hacer un mejor uso de la técnica de unir demandas (Glas, 2016: 67-70). Pero también puede resultar interesante aplicar por analogía soluciones probadas en otros sistemas, como el "US class action procedure», ya que permite que todas las partes con intereses comunes puedan estar representadas en el procedimiento (Kindt, 2018: 182; Sainati, 2015: 173 y ss.); o como las sentencias «aditivas» de principio dictadas por el Tribunal Constitucional italiano, que establecen un principio en favor de una categoría de sujetos lesionados e indican medidas de reparación al legislador (Sudre, 2016: 926 y 927); aunque siempre será necesaria una investigación adicional sobre su potencial eficacia en el plano internacional.

\section{LA RELACIÓN ENTRE EL ESTADO DEMANDADO Y EL TEDH: EL PRINCIPIO DE SUBSIDIARIEDAD}

El proceso de reforma del sistema del CEDH ha provocado un cambio de paradigma en la definición del principio de subsidiariedad, sobre el que se funda el procedimiento de sentencia piloto. Ya no se concibe tanto como un límite a la intervención del TEDH (López Guerra, 2014: 15), sino que se pone ahora el acento en que es a las autoridades nacionales a quien corresponde en primer lugar garantizar el disfrute efectivo de los derechos consagrados en el sistema del Convenio ${ }^{97}$, aunque sin olvidar que se trata de una «responsabilidad compartida $»^{98}$. Desde esta perspectiva, el procedimiento de sentencia piloto se configura precisamente como un medio a disposición del

96 Rapport explicatif, Protocole no 14 ..., cit., párrs. 10-12; Déclaration d'Interlaken, párr. dispositivo 1; Déclaration d'Izmir, Plan de Seguimiento, A; Déclaration de Brighton, párr. dispositivo 1; Déclaration de Bruxelles, párr. dispositivo 1; Déclaration de Copenhague, párr. dispositivo 1.

97 TEDH: Suivi d'Interlaken. Principe de Subsidiarité, Note du Jurisconsulte, 8 juillet 2010, párr. 2.

98 Déclaration de Copenhague, párr. 10. Véanse también Déclaration d'Interlaken, párr. del Preámbulo 1, 2 y 3; Déclaration d'Izmir, párr. del Preámbulo 5 y 6; Déclaration 
TEDH para ayudar al Estado a cumplir su papel de actor con responsabilidad principal en la protección de los derechos humanos (Kindt, 2017: 4).

Cierto es que el procedimiento de sentencia piloto fija el centro de gravedad en los Estados (Buyse, 2016: 102). Pone el peso de las decisiones en manos de las autoridades nacionales, que son las que intervienen en el diagnóstico del problema subyacente, identifican las medidas generales y resuelven los casos pendientes tras la renacionalización. Por otro lado, el aplazamiento permite que los Estados encuentren por sí mismos la solución haciendo que el Convenio se cumpla (Leach et al., 2010b: 30), los plazos suponen la interiorización de la idea de que los Estados deben adoptar a nivel nacional, en el menor tiempo posible, las medidas necesarias para conformarse al CEDH, y la renacionalización contribuye a que esta responsabilidad se haga efectiva.

Por su parte, el TEDH mantiene su rol de instancia subsidiaria de tutela (Queralt Jiménez, 2018: 422), aunque una relectura del art. 19 CEDH le permite verse a sí mismo como un asistente de los Estados en el cumplimiento de sus obligaciones en el marco del sistema del CEDH (Kindt, 2018: 178), dado que al fin y al cabo garantizar el disfrute de los derechos humanos consagrados en el Convenio es una responsabilidad compartida. Desde esta perspectiva, entiende que su función de tutela ante disfunciones crónicas no puede seguir consistiendo en identificar la misma violación estructural una y otra vez, ni tampoco en proveer una solución financiera individual a cada demandante. Debe entrar a valorar la propia compatibilidad de los sistemas normativos internos y políticas públicas respecto del estándar común europeo. Aunque este enfoque no es del todo nuevo (Queralt Jiménez, 2018: nota 32; Wallace, 2011:3-5 ${ }^{99}$, el procedimiento de sentencia piloto supone la formalización del control in abstracto, incidiendo en la dimensión constitucional del TEDH, y esto sin que se haya producido ningún cambio normativo convencional (Queralt Jiménez, 2018: 402 y 403; Sadurski, 2009: 422).

Sobre la base de esta responsabilidad compartida, el TEDH, para hacer frente a la disfunción crónica identificada, se involucra también en el proceso de ejecución de sus sentencias piloto, pero desde un planteamiento

de Brighton, párr. dispositivos 3 y 4; Déclaration de Bruxelles, párr. del Preámbulo 7; Déclaration de Copenhague, párr. 6 a 11.

99 Como ejemplos de sentencia europeas in abstracto, véanse Klass and Others $v$. Germany, judgment, 6 de septiembre de 1978, 2 EHRR 214; Marchx v. Belgium, judgment, 13 de junio de 1979, 2 EHRR 330; Norris v. Ireland, judgment, 26 de octubre de 1988, 13 EHRR 186; Dudgeon v. United Kingdom, judgment, 23 de septiembre de 1981, 4 EHRR 149; Driza v. Albania, judgment, 13 de noviembre de 2007, 49 EHRR 31. 
cooperativo $^{100}$, más que a través del recurso a la aplicación de sanciones o a la resolución a través de la imposición (Leach et al., 2010b: 32). Desde esta perspectiva, y aunque no se trata tampoco de un fenómeno totalmente novedoso, el TEDH «indica» en el fallo de la sentencia piloto el tipo de medidas correctoras (reparadoras y/o preventivas) que «deben» ser adoptadas por el Estado, pero dejándole un cierto margen de actuación. De este modo, el procedimiento de sentencia piloto «ha abierto la vía a la generalización del poder de recomendación del Tribunal y a su extensión sobre todo en el ámbito de las medidas generales» (Lambert Abdelgawad, 2007: 366). Esta práctica se ha criticado en la medida en que supone que el TEDH asuma no ya el papel de legislador negativo propio de los tribunales constitucionales continentales, sino de legislador positivo, no permitiendo siguiera valorar la oportunidad política (interna) de adoptar determinadas políticas públicas o determinadas normas internas (Fyrnys, 2011: 1233). En su defensa, se ha dicho que la alternativa al procedimiento de sentencia piloto, esto es, la condena en todas las sentencias pendientes, parece menos respetuosa con la soberanía nacional.

Dentro de este planteamiento cooperativo, el TEDH se reserva, no obstante, una cierta capacidad "persuasiva» (Leach et al., 2010b: 31). Así, puede «recompensar el esfuerzo» del Estado declarando la renacionalización de todas las demandas pendientes, dándole la oportunidad de poner fin a la violación del CEDH en lugar de condenarle en sucesivas ocasiones. Esta persuasión adquiere, no obstante, en ocasiones ciertos tintes conminatorios. Al fin y al cabo, el procedimiento de sentencia piloto se configura como una medida de último recurso, cuando otras vías más suaves han sido utilizadas para persuadir al Estado y no han funcionado (Garlicki, 2007: 190; Buyse, 2016: 112). Desde este otro enfoque, el TEDH puede incorporar en el fallo de la sentencia piloto un plazo para su ejecución. Otros elementos, como la reserva de la decisión de la satisfacción equitativa o el aplazamiento, también permiten ejercer una presión continua sobre el Estado, por ejemplo, recordándole que en cualquier momento el tribunal puede decidir sobre la satisfacción equitativa o retomar el examen de las demandas aplazadas ${ }^{101}$. Pero, sobre todo, la sentencia piloto no es una mera recomendación, sino un mandato, al menos por lo que se

${ }^{100}$ El juez Zupancic reconoce que la sentencia piloto supone decirle a Polonia: «Miren, ustedes tiene que resolver un grave problema y preferimos que lo resuelvan en casa... Si ello puede ayudarles, vamos a indicarles cuáles son para nosotros las normas mínimas a tomar en consideración a tal efecto".

101 Rumpf c. Alemnia, cit., párr. 75; Vassilios Athanassiou y otros c. Grecia, cit., párr. 58; TEDH, La procédure de l'arrêt pilote, cit., párr. 5; Queralt Jiménez, 2018: 415 y 422. 
refiere a los componentes incluidos en su parte operativa (Garlicki, 2007: 185; Haider, 2013: 45).

Pero el principio de subsidiariedad también se encuentra en la base de la decisión del tribunal de «lavarse las manos» en el procedimiento de sentencia piloto Ivanov c. Ucrania (Kurban, 2016: 769). Al adoptar, en el asunto Burmych y otros c. Ucrania, sobre esta base «un planteamiento político, eliminándose a sí mismo y a los demandantes de la ecuación» (Kindt, 2018: 188), el TEDH vendría de hecho a cerrar sus puertas a las víctimas de violaciones sistemáticas y graves (Kurban, 2016: 768) y a recompensar el incumplimiento, al eliminar su presión sobre el Estado - que, en cambio, una nueva condena hubiera incrementado-, lo que podría tener el perverso resultado de animar a los Estados a no ejecutar las sentencias piloto (Kindt, 2018: 186 y 187).

El procedimiento de sentencia piloto se revela, por tanto, inadecuado frente a incumplimientos persistentes. Algunas voces reclaman que el TEDH disponga en su «arsenal» del poder de imponer sanciones o multas de considerable cuantía frente a este tipo de incumplimientos (Susi, 2012: 413; o Fikfak, 2018). Pero esto requeriría una reforma mucho más estructural del TEDH y de su autoridad que la que se acaba de enfrentar.

\section{EL REPARTO DE COMPETENCIAS ENTRE EL COMITÉ DE MINISTROS Y EL TEDH: EL CONTROL DE LA EJECUCIÓN DE LAS SENTENCIAS PILOTO}

Del análisis que venimos realizando se deduce claramente que el procedimiento de sentencia piloto modifica el modelo de ejecución de las sentencias del TEDH previsto por el CEDH, que pivotaba hasta ahora en torno al Estado condenado, a quien corresponde la obligación de conformarse con las sentencias definitivas del TEDH, poniendo fin a la violación constatada y eliminado todas sus consecuencias (art. 46.1 CEDH), y al Comité de Ministros, "que velará por su ejecución» (art. 46.2 CEDH). El procedimiento de sentencia piloto incorpora en este modelo al TEDH al otorgarle un papel activo en la fase de ejecución de la sentencia piloto, lo que, como el propio TEDH reconoce, suponer dar una nueva dimensión a los roles respectivos del tribunal y del Comité de Ministros previstos en el Convenio ${ }^{102}$.

Para algunos, se rompería de este modo el equilibrio en el reparto horizontal de funciones establecida por el Convenio entre ambos órganos ${ }^{103}$. Para

102 Burmych et autres c. Ukraine, cit., párr. 141.

103 Hutten-Czapska c. Polonia, cit., opinión individual del juez Zagrebelsky; Lambert Abdelgawad, 2007: 366 y 368; Fyrnys, 2011: 1233; Queralt Jiménez, 2018: 421; Szymczak, 2011: 284; Wallace, 2011: 5. 
otros, no se puede olvidar que esta extralimitación se produce con la cobertura institucional del Comité de Ministros, en particular a través de la Res(2004)3 (Fyrnys, 2011: 1233; Queralt Jiménez, 2018: 421). Para el tribunal, en todo caso se ha hecho «en el respecto de las atribuciones respectivas de los órganos del Convenio» ${ }^{104}$.

Al fin y al cabo, «de conformidad con el art. $46 \$ 2$ [...], corresponde al Comité de Ministros apreciar las medidas generales e individuales adoptadas por el Estado y su ejecución» también en el procedimiento de sentencia piloto $^{105}$, aunque en este marco contaría además con la ayuda del TEDH (Wallace, 2011: 5). A diferencia del órgano político, la función del tribunal sería de naturaleza más puntual, ya que la abordaría «cuando examina asuntos que siguen a una sentencia piloto», y consistiría además en una evaluación más general o global, encaminada a "decidir si, a la vista de las medidas adoptadas por el Estado para remediar la violación constatada, la cuestión nuclear del litigio ha sido resuelta de conformidad con el art. 37 del Convenio y si procede continuar con el procedimiento de sentencia piloto» ${ }^{106}$. De este modo, no siempre se ha dado una identidad temporal en el período de ejercicio de estas funciones por ambos órganos ${ }^{107}$. La intervención del TEDH en la fase de ejecución tendría además un efecto principalmente interpretativo al contribuir a esclarecer el significado preciso de la sentencia piloto (Di Marco, 2016: 900), lo que poseería una doble virtualidad: prevenir que las partes soliciten la remisión a la Gran Sala a efectos interpretativos (art. $43 \mathrm{CEDH}$ ), que en ocasiones se ha utilizado de manera dilatoria (Di Marco, 2016: 900), pero también evitar que el Comité de Ministros pueda activar el recurso interpretativo previsto en el nuevo art. 46.3 CEDH.

Este nuevo reparto de competencias se fundaría, por tanto, en la noción de responsabilidad compartida, como también la decisión del Comité de Ministros de dar prioridad al seguimiento de las sentencias piloto a través

104 Association des propriétaires fonciers de Eódź et autres, cit., párr. 45.

105 Broniowski c. Polonia (règlement amiable), cit., párr. 42; Hutten-Czapska c. Pologne (règlement amiable) y Suljagić c. Bosnia-Herzegovina, cit., párr. 61; Association des propriétaires fonciers de Eódź et autres, cit., párr. 45.

106 Wolkenberg et autres, cit., párr. 77; E.G. c. Pologne et 175 autres affaires de la rivière Boug, cit., párrs. 25-29; y Association des propriétaires fonciers de Eódź et autres, cit., párr. 45.

107 Como vimos, mientras en algún caso el Comité de Ministros ha seguido supervisando la ejecución de la sentencia piloto tiempo después de la decisión del tribunal de cerrar el procedimiento (por ejemplo, en procedimiento Broniowski), en otros la decisión del tribunal ha sido posterior a la resolución final del Comité de Ministros (Kuric y otros c. Eslovenia, ad exemplum). 
de un "procedimiento continuo» ("procédure soutenue»), que implica un seguimiento más proactivo que si el caso quedara sometido al procedimiento estándar ${ }^{108}$. Sobre la base de esta misma noción, el Protocolo $n^{\circ} 14$ ha puesto en manos del Comité de Ministros un instrumento adicional de presión frente a Estados recalcitrantes a la hora de ejecutar cualquier tipo de sentencias del tribunal —y por tanto, también las sentencias piloto (Kindt, 2018: 180)—: el recurso por incumplimiento ante el TEDH del art. 46.4 y 5 CEDH. Su utilización tiene como resultado la obtención de una sentencia del tribunal, que, aunque declarativa ${ }^{109}$, también es obligatoria, con la consiguiente responsabilidad internacional que podría derivarse de su incumplimiento.

El procedimiento de sentencia piloto, pero también el recurso por incumplimiento, suponen, por tanto, una cierta judicialización de la función de supervisión de la ejecución de las sentencias ${ }^{110}$. Para algunos podría considerarse incluso una suerte de recuperación de las funciones judiciales por parte del TEDH, ya que «determinar las consecuencias jurídicas de una violación del derecho está en el núcleo mismo de la función judicial» ${ }^{111}$. Pero si bien formalmente parece que queda reforzado el carácter supremo del TEDH (Lambert Abdelgawad, 2007: 380), las medidas de sanción a los Estados incumplidores no tienen más recorrido, resultando claramente insuficientes frente a Estados recalcitrantes, como, por otra parte, pone claramente de manifiesto el asunto Ivanov c. Ucrania o la primera sentencia dictada por el TEDH en un procedimiento de incumplimiento en el asunto Ilgar Mammadov c. Azerbaiyán.

108 Committee, Information Document, CM/Inf/DH (2010) 45 final, 7 de diciembre de 2010, párr I(10); Committee, Information Document, CM/Inf/DH(2010)37, 6 de septiembre de 2010, párrs. 6, 8, 20; Règles du Comité des Ministres pour la surveillance de l'exécution des arrêts et des termes des règlements amiables, Règles $\mathrm{n}^{\circ \mathrm{s}} 4 \mathrm{y} 7$. Véanse también Glas, 2016: 59; Szymczak, 2011: 284.

109 Este recurso por incumplimiento no tiene por finalidad reabrir ante el tribunal la cuestión de la violación ya resuelta en la primera sentencia, ni tampoco la imposición de una pena financiera (Rapport explicatif, Protocole $n^{\circ} 14 \ldots$, cit., párrs. 99 y 100), al rechazarse la propuesta inicial de la Asamblea Parlamentaria de imponer una multa diaria en caso de retraso en la ejecución de las sentencias (Résolution 1226 (2000) y Recommandation 1477(2000) de la Asamblea Parlamentaria). De conformidad con el art. 46.4 y 5, se limita a concluir si se ha producido o no una violación del art. 46.1 CEDH.

110 TEDH, Procédure fondée sur l'article $46 \$ 5$ dans l'affaire Ilgar Mammadov c. Azerbaïdjan [GC] (no 15172/13), arrêt, 29 de mayo de 2019, opinión concordante de la juez Motoc, párr. 5; Fyrnys, 2011: 353.

111 Ilgar Mammadov c. Azerbaiyán, opinión concordante du juge Wojtyczek, párrs. 8 y 9. 
Las dos alternativas a la solución adoptada por el TEDH en el caso Burmych y otros c. Ucrania propuestas por los jueces disidentes confirman este extremo ${ }^{112}$. Frente al incumplimiento persistente de Ucrania, proponían, en primer lugar, retomar el examen individual de cada caso, aunque por el Comité de tres jueces al existir una jurisprudencia consolidada del TEDH, con un menor coste en recursos. La segunda alternativa propuesta era el uso del recurso por incumplimiento por parte del Comité de Ministros, lo que conllevaría una condena al Estado por la situación de incumplimiento considerada en su conjunto. Sin menospreciar la presión que para el Estado suponen nuevas condenas, de cara al disfrute efectivo de los derechos humanos resultan soluciones claramente insuficientes.

\section{CONCLUSIONES}

El procedimiento de sentencia piloto es, como hemos visto, un mecanismo transformador de primer orden de la función del TEDH, que es llamado a adoptar un papel más activo, pero también del sistema del CEDH en su conjunto. Este procedimiento permite al tribunal tomar la decisión de tramitar una demanda como piloto como vía para administrar justicia más allá del caso concreto seleccionado, anticipándose incluso a futuras demandas, lo que incide además directamente en su relación con los demandantes afectados, que son tratados de manera diferenciada en atención a su condición de demandantes piloto o demandantes clones. Los controles in abstracto que realiza y su participación en la fase de ejecución de las sentencias piloto también afecta a su relación con el Estado condenado y con el Comité de Ministros, potenciando la dimensión constitucional del TEDH e incidiendo en el reparto de competencias dentro del sistema del Convenio. La relación del TEDH con los demandantes, con el Estado condenado y con el Comité de Ministros responde, por tanto, en el procedimiento de sentencia piloto a unos parámetros diferentes a los tradicionalmente establecidos.

Precisamente este particular diseño del procedimiento de sentencia piloto que permite caracterizarlo de transformador ha contribuido a generar en la práctica importantes beneficios para el sistema del Convenio. Ha permitido al TEDH enfrentar los contenciosos repetitivos que lastraban su carga de trabajo y prevenir nuevas demandas. Cierto es que no es un instrumento para afrontar «violaciones graves de los derechos humanos», aunque esos problemas sistémicos o estructurales en los que trae causa este tipo de contenciosos puedan

112 Burmych et autres c. Ukraine, cit., opinión disidente, párrs. 20 y 24. 
en algunos casos generar graves violaciones de los derechos humanos y/o del Estado de derecho. Su función consiste en evitar al TEDH tener que repetir hasta el infinito el mismo mensaje, lo que ha provocado una reducción importante de su carga de trabajo y le ha permitido centrarse en resolver cuestiones relevantes de interés general.

Por otro lado, la visión colaborativa que configura la función del TEDH en la fase de ejecución de la sentencia piloto se ha traducido en valiosas indicaciones para los Estados en cuanto a las medidas generales a adoptar con el fin de corregir el problema sistémico o estructural, pero también para el Comité de Ministros sin necesidad de que este ponga formalmente en marcha los nuevos mecanismos introducidos en el art. $46 \mathrm{CEDH}$, lo que ha contribuido a la mejora de la ejecución de las sentencias del TEDH. Además, siempre que los Estados se han mostrado colaboradores, se ha agilizado la reparación íntegra a los demandantes y demás víctimas, que no han tenido siquiera que acudir al tribunal, logrando el efecto preventivo que se pretendió al diseñar este procedimiento. No cabe duda, por tanto, de que nos hallamos ante un procedimiento que con carácter general se puede decir que responde a los objetivos para los que fue creado, cumpliéndolos en buena medida.

Sus ventajas y el alto grado de éxito en el uso de este procedimiento no deben, sin embargo, hacernos perder de vista que se ha revelado inadecuado para afrontar situaciones en las que el Estado no podía o no quería enfrentar el problema sistémico. A pesar de que este procedimiento reserva al TEDH una cierta capacidad persuasiva teñida con ciertos tintes conminatorios, su éxito reposa en gran medida en la actitud del Estado condenado. Este escenario nos enfrenta a dos situaciones. La primera es que el procedimiento de sentencia piloto debe utilizarse únicamente después de una valoración exhaustiva de la complejidad del asunto y de la voluntad y compromiso del Estado implicado. Su uso debiera ser, por tanto, excepcional. Esta opción parece haber sido finalmente adoptada por el TEDH, dado que lleva varios años sin iniciar un nuevo procedimiento de sentencia piloto.

En segundo lugar, es necesario reforzar el mecanismo de ejecución del $\mathrm{CEDH}$, ya que, aunque el sistema se ha dotado del procedimiento de sentencia piloto y del nuevo recurso de incumplimiento, sigue careciendo de capacidad coercitiva real sobre el Estado recalcitrante, más allá de la acumulación de condenas. Este es un reto a futuro que no puede dejarse por mucho más tiempo, porque ataca a la línea de flotación del sistema: la autoridad del tribunal.

Además de no resolver esta problemática, contribuyendo en cambio a hacerla más evidente, el procedimiento de sentencia piloto puede llegar a tener un efecto realmente perverso sobre el conjunto del sistema del Convenio, ya que viene a menoscabar de manera preocupante el derecho de recurso individual ante el TEDH. Esto exige ciertos reajustes, lo que, unido a la necesidad 
de repensar la capacidad coercitiva del tribunal, nos sitúa ante una nueva reforma, si bien no integral como la recientemente abordada, sí de calado, que no debiera ser aprovechada para menoscabar la piedra angular del sistema del $\mathrm{CEDH}$.

\section{Bibliografía}

Abrisketa Uriarte, J. (2013). Las sentencias piloto: el Tribunal Europeo de Derechos Humanos, de juez a legislador. Revista Española de Derecho Internacional, 65 (1), 73-99.

Acosta Alvarado, P. A. (2008). Tribunal Europeo y Corte Interamericana de derechos humanos: ¿Escenarios idóneos para la garantía del derecho de acceso a la justicia internacional? [ebook]. Universidad Externado de Colombia.

Buyse, A. (2016). Flying or landing? The pilot judgment procedure in the changing European human rights architecture. En O. Mjöll Arnardóttir y O. Buyse (eds.). Shifting Centres of Gravity in Human Rights Protection. Rethinking Relations between the ECHR, EU, and National Legal Orders (pp. 101-115). London: Routledge.

Czepek, J. (2018). The Application of the Pilot Judgment Procedure and Other Forms of Handing Large-Scale Dysfunctions in the Case Law of the European Court of Human Rights. International Community Law Review, 20, 347-373. Disponible en: https://doi.org/10.1163/18719732-12341379.

Di Marco, A. (2016). L'État face aux arrêts pilotes de la Cour Européenne des Droits de l'Homme. Revue Trimestrielle des Droits de l'Homme, 108, 887-914.

Ducoulombier, P. (2011). Arrêts pilotes et efficacité des nouveaux recours internes. En E. Lambert-Abdelgawad y P. Dourneau-Josette (dirs.). Le filtrage des affaires à Strasbourg: l'examen de la recevabilité par la Cour européenne des droits de l'homme (pp. 255-292). Strasbourg: Éditions du Conseil de l'Europe. Disponible en: https://doi.org/10.3917/europ.dour.2013.01.0255.

Fikfak, V. (2018), Changing State Behaviour: Damages before the European Court of Human Rights. European Journal of International Law, 29 (04), 1091-1125. Disponible en: https://doi.org/10.1093/ejil/chy064.

Fyrnys, M. (2011). Expanding Competences by Judicial Lawmaking: The Pilot Judgment Procedure of the European Court of Human Rights. German Law Journal, 12 (5), 1231-1259. Disponible en: https://doi.org/10.1017/ S2071832200017284.

Garlicki, L. (2007). Broniowski and After: On the Dual Nature of 'Pilot Judgments'. En L. Caflisch, J. Callewaert, R. Liddell, P. Mahoney y M. Villiger (eds.). Liber Amicorum Luzius Wildhaber: Human Rights - Strasbourg Views (pp. 177-192). Kehl: Engel. 
Gerards, J. H. y Glass, L. R. (2017). Access to justice in the European Convention on Human Rights System. Netherlands Quarterly of Human Rights, 35 (1), 11-30. Disponible en: https://doi.org/10.1177/0924051917693988.

Glas, L. (2016). The Functioning of the Pilot-Judgment Procedure of the European Court of Human Rights in Practice. Netherlands Quarterly of Human Rights, 34 (1), 41-70. Disponible en: https://doi.org/10.1177/016934411603400104.

Haider, D. (2013). The Pilot-Judgement Procedure of the European Court of Human Rights. Leiden: Martinus Nijhoff Publishers. Disponible en: https://doi. org/10.1163/9789004246447.

Kindt, E. (2017). Non-execution of a pilot judgment: ECtHR passes the buck to the Committee of Ministers in Burmych and others v. Ukraine. Strasbourg Observers [blog], 26-10-2017. Disponible en: https://bit.ly/2T0OA1V.

Kindt, E. (2018). Giving up on individual justice? The effect of state non-execution of a pilot judgment on victims. Netherlands Quarterly of Human Rights, 36 (3), 173-188. Disponible en: https://doi.org/10.1177/0924051918785005.

Kurban, D. (2016). Forsaking Individual Justice: The Implications of the European Court of Human Rights' Pilot Judgment Procedure for Victims of Gross and Systematic Violations. Human Rights Law Review, 16 (4), 731-769. Disponible en: https://doi.org/10.1093/hrlr/ngw032.

Lambert Abdelgawad, E. (2007). El Tribunal Europeo de Derechos Humanos y la técnica de las sentencias piloto: una pequeña revolución en marcha en Estrasburgo. Revista de Derecho Politico, 69, 355-383. Disponible en: https://doi. org/10.5944/rdp.69.2007.9026.

Leach, P., Hardman, H. y Stephenson, S. (2010a). Can the European Court's Pilot Judgment Procedure Help Resolve Systemic Human Rights Violations? Burdov and the Failure to Implement Domestic Court Decisions in Russia. Human Rights Law Review, 10 (2), 346-359. Disponible en: https://doi.org/10.1093/ hrlr/ngq011.

Leach, P., Hardman, H., Stephenson, S. y Blitz, B. K. (2010b). Responding to systemic human rights violations: an analysis of pilot judgments of the European Court of Human Rights and their impact at national level. Antwerp; Oxford; Portland: Intersentia.

López Guerra, L. M. (2014). Los Protocolos de reforma no 15 y 16 al Convenio Europeo de Derechos Humanos. Civitas. Revista Española de Derecho Europeo, 49, 11-29.

Queralt Jiménez, A. (2018). Las sentencias piloto como ejemplo paradigmático de la transformación del Tribunal Europeo de Derechos Humanos. Universidad Nacional de Educación a Distancia. Teoría y Realidad Constitucional, 42, 395-424. Disponible en: https://doi.org/10.5944/trc.42.2018.23637.

Quispe Remón, F. (2018). Acceso a la justicia y Objetivos del Desarrollo Sostenible. En C. M. Díaz Barrado y C. R. Fernández Liesa (dirs). Objetivos de desarrollo sostenible y derechos humanos: paz, justicia e instituciones sólidas/derechos humanos y empresas (pp. 235-248). Madrid: Universidad Carlos III de Madrid. 
Rosu, A.-C. (2014). Property Restitution in Romania. Global Economic Observer, 2 (2), 37-41.

Sadurski, W. (2009). Partnering with Strasbourg: Constitutionalism of the European Court of Human Rights, the Accession of Central and East European States to the Council of Europe, and the Idea of Pilot Judgments. Human Rights Law Review, 9 (3), 397-453. Disponible en: https://doi.org/10.1093/hrlr/ngp011.

Sainati, T. (2015). Human Rights Class Actions: Rethinking the Pilot-Judgment Procedure at the European Court of Human Rights. Harvard International Law Journal, 56 (1), 147-205.

Sardaro, P. (2005). The Right of Individual Petition to the European Court. En P. Lemmens y W. Vandenhole. Protocol no 14 ant the Reform of the European Court of Human Rights (pp. 45-47). Cambridge, U. K.: Intersentia Publishers.

Sudre, Fr. (2016). L'effectivité des arrêts de la Cour européenne des droits de l'homme. Revue Trimestrielle Droits de l'Homme, 108, 917-947.

Susi, M. (2012). The Definition of a Structural Problem in the Case-Law of the European Court of Human Rights since 2010. German Yearbook of International Law, 55, 385-417.

Szymczak, D. (2011). Le droit à indemnisation dans le cadre des "procédures pilotes». En J-F. Flauss y E. Lambert (dirs.). La pratique d'indemnisation par la cour européenne des droits de l'homme (pp. 275-295). Brusselles: Bruylant.

Ulfstein, G. y Zimmermann, A. (2018). Certiorari through the Back Door: The Judgment by the European Court of Human Rights in Burmyuch and Others v. Ukraine in Perspective. Kolleg-Forschergruppe. Working Paper Series, 13. Disponible en: https://doi.org/10.2139/ssrn.3150341.

Von Bogdandy, A. y Ioannidis, M. (2014). La deficiencia sistémica en el Estado de Derecho, qué es, qué se ha hecho y qué se puede hacer. Revista de Estudios Políticos, 165, 59-96.

Wallace, S. (2011). Much ado about nothing? The pilot judgment procedure at the European Court of Human Rights. European Human Rights Law Review, 71-81.

Wildhaber, L. (2009). Pilot Judgments in Cases of Structural or Systemic Problems on the National Level. En U. Deutsch y R. Wolfrum (eds.). The European Court of Human Rights Overwhelmed by Applications: Problems and Possible Solutions (pp. 69-75). Beiträge zum ausländischen öffentlichen Recht und Völkerrecht (Veröffentlichungen des Max-Planck-Instituts für ausländisches öffentliches Recht und Völkerrecht), 205. Berlin; Heidelberg: Springer. 